\title{
Immunosuppressive Features of the Microenvironment in Lymph Nodes Granulomas from Tuberculosis and HIV-Co-Infected Patients
}

Senait Ashenafi, ${ }^{* \dagger}$ Jagadeeswara Rao Muvva, ${ }^{*}$ Akhirunnesa Mily, ${ }^{*}$ Johanna Snäll, ${ }^{*}$ Martha Zewdie, ${ }^{\ddagger}$ Menberework Chanyalew, Anders Rehn, * Sayma Rahman, ${ }^{*}$ Getachew Aseffa, ${ }^{\S}$ Amsalu Bekele, ${ }^{\top}$ Getachew Aderaye, "Beede Lema, Mattias Svensson, ${ }^{*}$ and Susanna Brighenti*

From the Center for Infectious Medicine, ${ }^{*}$ Department of Medicine, ANA Futura, Karolinska Institutet, Huddinge, Sweden; Departments of Pathology, ${ }^{\dagger}$ Radiology, ${ }^{\S}$ Internal Medicine, " and Surgery," Tikur Anbessa Specialized Hospital and Addis Ababa University, College of Health Sciences, Addis Ababa, Ethiopia; and Armauer Hansen Research Institute, ${ }^{\ddagger}$ Addis Ababa, Ethiopia

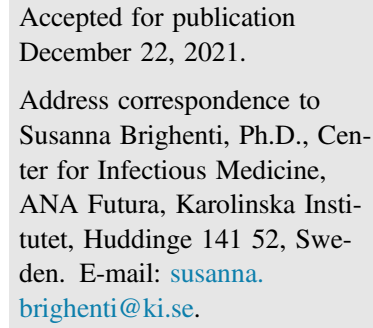

\begin{abstract}
Tuberculosis (TB) and HIV co-infection claims many lives every year. This study assessed immune responses in Mycobacterium tuberculosis-infected lymph node tissues from HIV-negative and HIV-positive patients compared with the peripheral circulation with a focus on myeloid cells and the cell-signaling enzymes, inducible nitric oxide synthase, and arginase (Arg)-1. Methods included immunohistochemistry or confocal microscopy and computerized image analyses, quantitative real-time PCR, multiplex Luminex, and flow cytometry. These findings indicate enhanced chronic inflammation and immune activation in TB/ HIV co-infection but also enhanced immunosuppressive responses. Poorly formed necrotic TB granulomas with a high expression of $M$. tuberculosis antigens were elevated in TB/HIV-co-infected lymph nodes, and inducible nitric oxide synthase and Arg-1 expression was significantly higher in TB/HIV-co-infected compared with HIV-negative TB or control tissues. High Arg-1 expression was found in myeloid cells with a phenotype characteristic of myeloid-derived suppressor cells (MDCS) that were particularly abundant in TB/HIV-co-infected tissues. Accordingly, Lin $^{-} / \mathrm{HLA}^{-D R^{\text {low }} / \text { int }} / \mathrm{CD} 33^{+} / \mathrm{CD} 11 \mathrm{~b}^{+} / \mathrm{CD} 15^{+}$granulocytic myeloid-derived suppressor cells were significantly elevated in blood samples from TB/HIV-co-infected patients. CD15 ${ }^{+}$myeloid-derived suppressor cells correlated with plasma HIV viral load and M. tuberculosis antigen load in tissue but were inversely associated with peripheral CD4 T-cells counts. Enhanced chronic inflammation driven by M. tuberculosis and HIV co-infection may promote Arg-1-expressing MDSCs at the site of infection thereby advancing TB disease progression. (Am J Pathol 2022, 192: 653-670; https:// doi.org/10.1016/j.ajpath.2021.12.013)
\end{abstract}

HIV infection is a major comorbidity of tuberculosis (TB) that is present in $15 \%$ of all TB deaths and currently affects approximately 15 million people around the world. ${ }^{1}$ Cellular immunity is gradually destroyed in Mycobacterium tuberculosis as well as HIV infection, manifesting in severe depletion of $\mathrm{CD}^{+} \mathrm{T}$ cells and dysfunctional activity of macrophages and dendritic cells (DCs). Activation of macrophages and DCs results in elaboration of proinflammatory cytokines, induction of antimicrobial effector pathways, and priming of specific Th1 and cytolytic effector T cells that are required to kill intracellular $M$. tuberculosis. Th1 cytokines, such as IL-1 $\beta$, tumor necrosis factor- $\alpha$, and interferon
(IFN)- $\gamma$, are instrumental in activation, recruitment, and organization of immune cells at the site of M. tuberculosis infection, which results in TB granuloma formation. TB granulomas are a hallmark of human TB and defined as dynamic cellular clusters of $M$. tuberculosis-infected

\footnotetext{
Supported by Swedish Heart and Lung Foundation grants 2019-0299 and 2019-0302 (S.B.), Swedish Research Council grants 2019-01744 and 201904720 (S.B.), and the Foundation to Prevent Antibiotic Resistance. The funders had no role in study design, experimental works, data analysis, decision to publish, or preparation of the manuscript.

Disclosures: None declared.
} 
macrophages, which are surrounded by a peripheral cuff of $\mathrm{T}$ cells and other immune cells. ${ }^{2,3}$ Although productive granulomas are considered to isolate and limit spread of $M$. tuberculosis infection, ${ }^{4}$ their formation may fail in individuals with a compromised immune system, including TB/HIV-co-infected patients. ${ }^{5}$ This process is likely dependent on the degree of HIV-induced immunosuppression from mild or moderate to more advanced immunosuppression characterized by reduced cell recruitment to the TB granuloma and release of large numbers of M. tuberculosis bacilli. ${ }^{6}$

Myeloid cells have a central role in TB and HIV infection, including a highly heterogenous population of mononuclear myeloid cells, such as not only monocytes, macrophages, and DCs but also granulocytic myeloid cells and myeloid-derived suppressor cells (MDSCs). ${ }^{7}$ MDSCs are pathologically activated myeloid cells with immunosuppressive traits that were originally described in the tumor immunology field, ${ }^{7}$ although these cells are also involved in chronic inflammatory conditions, including $\mathrm{TB}^{8}$ and HIV infection. ${ }^{9}$ MDSCs comprise a heterogenous population of monocytic or granulocytic subsets that also contain immature progenitors or early-stage MDSCs. ${ }^{10}$ Such myeloid cell subsets impair protective $\mathrm{T}$-cell responses and may contribute to the inability to eradicate these intracellular pathogens at mucosal sites and instead promote persistent infection. $^{11}$

It has been proposed that amino acid metabolism in myeloid cells provides important cues that regulate both innate and adaptive immune responses in different diseases. ${ }^{12}$ Mouse data suggest that classically activated proinflammatory macrophages (M1) produce nitric oxide (NO) and engage in bacterial killing, as opposed to alternatively activated anti-inflammatory macrophages (M2) that instead use arginase (Arg)-1 to induce fibrosis and prohealing responses. ${ }^{13,14}$ The inducible nitric oxide (iNOS) and Arg-1 regulate L-arginine levels in tissue by competing for cellular L-arginine, which is the substrate for both of these enzymes. ${ }^{15}$ M1 or M2 polarized macrophages stimulate Th1/cellular or Th2/antibody responses, respectively, which further amplify M1/M2 responses via production of IFN- $\gamma$ or IL- $4 .{ }^{16}$ Macrophages and DCs could also express the immunosuppressive enzyme indoleamine 2,3-dioxygenase (IDO) that is involved in tryptophan degradation, which may impair both innate and adaptive immune responses and thus assist microbes to escape eradication by the immune system. Tissue deprivation of L-arginine ${ }^{17}$ or tryptophan ${ }^{18}$ contributes to not only reduced proliferation and activation of $\mathrm{T}$ cells but also induction of regulatory $\mathrm{T}$ (Treg) cells. iNOS, Arg-1, and IDO are also factors implicated in MDSC-mediated immunosuppression, with the main target being effector T cells. ${ }^{7,19}$

TB/HIV co-infection often manifests as cervical lymphadenopathy, a finding that is also common in patients infected by TB or HIV alone. ${ }^{20}$ Accordingly, human lymph node tissue and blood from HIV-negative or HIV-positive TB-infected individuals were used to explore the effect of HIV co-infection in TB disease, particularly in TB granuloma formation. Specific subsets of myeloid cells and effector molecules as well as immunosuppressive pathways in the local environment of $M$. tuberculosis-infected lymph nodes were studied.

\section{Material and Methods}

\section{Study Participants and Diagnosis}

Participants were recruited at the Tikur Anbessa University Hospital in Addis Ababa, Ethiopia, after providing signed informed consent. Inclusion criteria were individuals $>18$ years of age who were HIV negative or HIV positive and had cervical lymph node enlargement and normal chest radiographic findings. Common clinical signs of TB infection, such as fever, sweating, or weight loss, were not used to stratify patients. Exclusion criteria were a history of previous TB or $>1$ week of antimicrobial chemotherapy, presence of pulmonary involvement consistent with TB, current or prior antiretroviral therapy, or lack of consent to HIV screening. Individuals with nonspecific reactive enlargement of the cervical lymph nodes with no signs of TB disease were recruited as controls. Ethical approval of the study was obtained from the national ethical review boards in Ethiopia and Sweden.

The study participants $(n=23)$ were divided into three groups: i) TB-single infection $(n=8)$, ii) TB/HIV coinfection $(n=8)$ and iii) HIV-negative controls $(n=3)$ and HIV-positive controls $(n=4)$ without TB disease (Table 1). TB diagnosis was based on a combination of histopathological evidence as well as clinical symptoms, including clinical response after completed anti-TB chemotherapy, which was defined as resolution of lymph node enlargement. TB-positive specimens $(n=16)$ revealed a granulomatous inflammation with epithelioid cell clusters, multinucleated giant cells, and caseating necrosis consistent with TB. The histologic features of TB-negative control lymph node samples $(n=7)$ typically demonstrated a reactive lymphoid hyperplasia with no granuloma formation.

\section{Clinical Samples and Procedures}

An excisional biopsy of a single enlarged cervical lymph node was obtained from each study participant, along with matched peripheral blood. Lymph node biopsy specimens were divided into two parts for future analysis: tissue fixed in formalin (Sigma-Aldrich, St. Louis, MO) and embedded in paraffin blocks and tissue preserved in RNAlater (BioRad, Hercules, CA) at $-85^{\circ} \mathrm{C}$. Hematoxylin and eosin (H\&E) (Sigma-Aldrich)-stained tissue sections were used for histopathological diagnosis of the lymph node samples. Plasma samples were used for HIV screening and determination of plasma HIV viral load, according to the national guidelines in Ethiopia. Blood chemistry analyses included assessment of hemoglobin, white blood cell counts , and 
Table 1 Clinical and Laboratory Characteristics of Study Participants

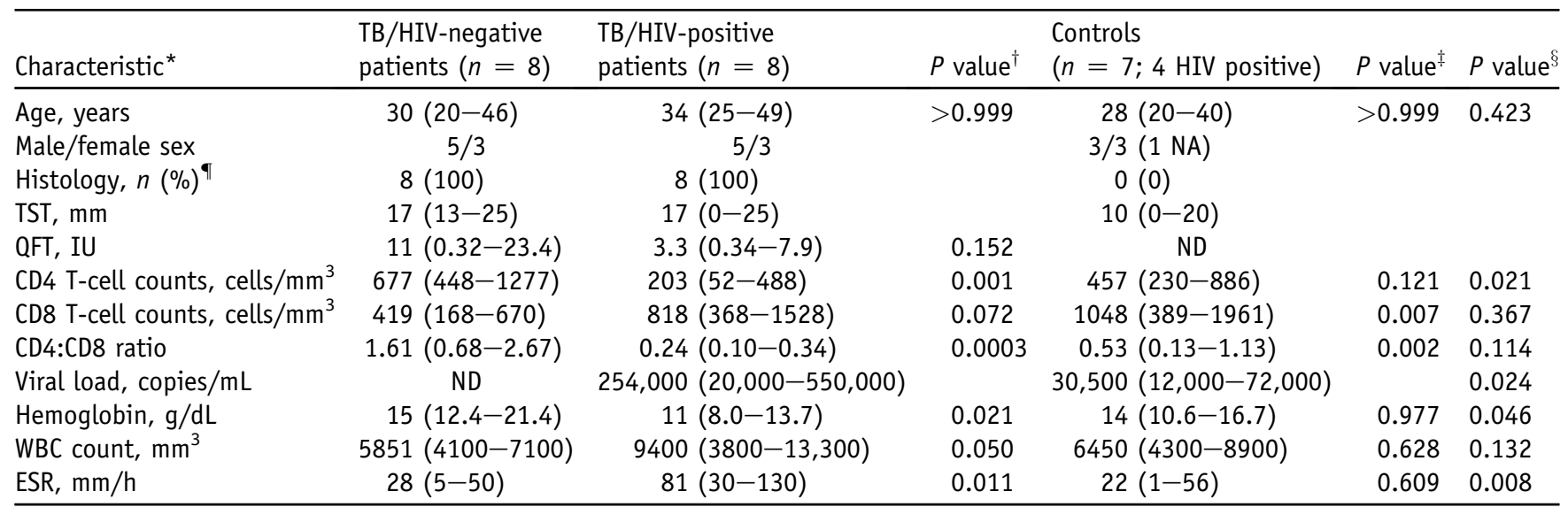

*Data are presented as mean (range) unless otherwise stated.

${ }^{\dagger}$ TB versus TB/HIV (Kruskal-Wallis test and Dunn posttest).

${ }^{\ddagger} T B$ versus controls (Kruskal-Wallis test and Dunn posttest).

${ }^{\S}$ TB/HIV versus controls (Kruskal-Wallis test and Dunn posttest).

"Histologically confirmed TB diagnosis of excisional biopsy of cervical lymph nodes showing typical morphological changes consistent with TB.

ESR, erythrocyte sedimentation rate; NA, not available; ND, not determined; QFT, QuantiFERON-TB Gold in-Tube; TB, tuberculosis; TST, tuberculin skin test; WBC, white blood cell.

erythrocyte sedimentation rate (ESR). Immunologic status was assessed using the tuberculin skin test (Statens Serum Institut, København, Denmark), whereas whole blood samples were used for peripheral CD4/CD8 T-cell counts (FACSCount; BD Biosciences, Franklin Lakes, NJ), and IFN- $\gamma$ production was measured with the QuantiFERONTB Gold In-Tube (Cellestis, Victoria, Australia) according to the manufacturer's instructions. A tuberculin skin test reaction measured at 48 to 72 hours after tuberculin (Statens Serum Institute) injection was considered a positive result when the transverse induration was $\geq 10 \mathrm{~mm}$ for $\mathrm{TB}$ and $\geq 5$ $\mathrm{mm}$ for TB/HIV-co-infected patients, whereas QuantiFERON-TB Gold In-Tube results above a cut-off of $0.35 \mathrm{IU} / \mathrm{mL}$ were considered positive.

\section{Antigen Retrieval in Paraffin-Embedded Lymph Node Tissues}

Formalin-fixed, paraffin-embedded tissue biopsy specimens were cut into 3 - $\mu$ m-thick sections and mounted on Superfrost Plus microscope slides (Thermo Fisher Scientific/ Menzel-Gläser, Waltham, MA). The tissue was deparaffinised using xylene (Thermo Fisher Scientific) for $3 \times 5$ minutes followed by rehydration with decreasing concentrations of ethanol (Solveco, Stockholm, Sweden) at $100 \%$ for $2 \times 5$ minutes, $95 \%$ for 5 minutes, and $70 \%$ for $5 \mathrm{mi}-$ nutes. Dewaxed slides were rinsed with Tris-buffered saline (TBS; Sigma-Aldrich), and endogenous peroxidase was blocked with $3 \% \mathrm{H}_{2} \mathrm{O}_{2}$ (Sigma) for 30 minutes before antigen retrieval was performed by microwave boiling in 0.01 mol/L citrate buffer ( $\mathrm{pH} \mathrm{6)}$ (Sigma-Aldrich) for $2 \times 2.5$ minutes. After cooling and washing in TBS, sectioned tissues were used for immunostains as described below.

\section{Immunostaining of Lymph Node Tissues}

Deparaffinized and rehydrated tissue sections were blocked for 30 minutes with $5 \%$ fetal calf serum (Sigma-Aldrich) in TBS before staining with immunohistochemistry (IHC) or immunofluorescence (IF). IHC was performed using the Vectastain Elite ABC-kit (Vector Laboratories, Burlingame, CA) as previously described, ${ }^{21-23}$ and positive stain was developed using a diaminobenzidine substrate (Vector Laboratories) and hematoxylin (Sigma-Aldrich) for nuclear counterstaining. For co-localization of immune molecules, 2- to 4-color stains were performed using indirect IF and DAPI mounting media (Vector Laboratories) for visualization of cell nuclei.

Primary antibodies used for IHC were monoclonal mouse anti-human CD3, CD4, and CD8 (BD Biosciences, San Jose, CA), CD20 and collagen type I (Abcam, Cambridge, UK), CD68 and CD163 (Novocastra, Leica Biosystems, Wetzlar, Germany), monoclonal rabbit anti-human CD11b and $\mathrm{CD} 11 \mathrm{c}$ (Abcam) and polyclonal rabbit anti-human iNOS (Thermo Fisher Scientific), Arg-1 and CD163 (GeneTex, Irvine, CA), and IDO-1 (Sigma). A polyclonal rabbit anti-M. tuberculosis bacteria antibody (Invitrogen, Waltham, MA) was used for detection of M. tuberculosis, whereas a polyclonal rabbit anti-HIV-1 p55+p24+p17 antibody (BIOSS Antibodies, Boston, MA) was used for detection of HIV antigens in tissue. Biotinylated secondary antibodies for IHC included goat anti-mouse IgG and swine anti-rabbit $\mathrm{F}(\mathrm{ab})_{2}$ from Dako Cytomation (Jena, Germany). Tissue sections stained with secondary antibodies only were used as negative controls. Primary antibodies used for IF were monoclonal mouse anti-human CD68, CD163, CD33, CD3, CD56, CD123 (Novocastra), CD15, CD20, MAC387 
Table 2 Histopathological Staging of Granulomas in Lymph Node Tuberculosis

\begin{tabular}{lll}
\hline Granuloma & Appearance & Necrosis \\
\hline Stage 1 & Irregular clusters of epithelioid cells interspersed with lymphocytes & Absent \\
Stage 2 & Epithelioid cell aggregates with well-defined borders & Absent or minimal necrotic areas \\
Stage 3 & Epithelioid cell aggregates with well-defined borders & Limited areas of central caseous necrosis \\
Stage 4 & Multicentric epithelioid cell clusters with irregular borders & Extensive areas of caseous necrosis \\
\hline
\end{tabular}

Lymph node granulomas were examined and graded using a modified version of a previously described histopathologic staging of mycobacterial granulomas in hematoxylin and eosin-stained tissue sections. ${ }^{24}$

(Dako), HLA-DR (Abcam), polyclonal rabbit anti-human iNOS (Thermo Fisher Scientific), Arg-1, and CD163 (GeneTex). Primary antibodies were added and incubated for 90 minutes (polyclonal antibodies) or overnight (monoclonal antibodies) in room temperature. Before addition of the secondary antibodies, tissue sections were washed with TBS and blocked for 30 minutes with $1 \%$ normal goat serum (Sigma-Aldrich) in TBS. The secondary antibodies were added and incubated for 30 minutes (IF) or 1 hour (IHC) in the dark. Fluorophore-conjugated secondary antibodies used for IF included goat anti-mouse IgG Alexa488, goat anti-mouse IgG2b Alexa/Fluor-555, and goat antimouse IgG1 Alexa/Fluor-647 (Invitrogen), goat pAb to Ms IgM Daylight-488 (Abcam), as well as goat anti-rabbit IgG Alexa-594 (Molecular Probes, Eugene, OR).

\section{Quantitative Image Analysis of Lymph Node Tissues}

Acquired computerized image analysis was used to quantify IHC staining by transferring digital images of the stained tissue samples from a DMR-X microscope to a computerized Quantimet 5501W image analyzer (Leica Microsystems). Positive immunostaining was quantified at the single-cell level in 10 to 50 high-power fields with a mean total area of $1.5 \times 10^{5} \mu \mathrm{m} /$ field, using the Qwin 550 v.2 software program (Leica Imaging Systems, Wetzlar, Germany). Approximately 50 microscopy fields were assessed in TB- and TB/HIV-infected lymph nodes and approximately 10 to 30 fields for control lymph nodes, which were mostly smaller compared with the TB-infected lymph nodes. Protein expression was determined as the percentage positive area of the total relevant cell area defined as the nucleated and cytoplasmic area within the tissue biopsy, excluding fibrotic and necrotic tissue areas. Occasionally, positive immunostaining determined inside the TB granulomas was compared with tissue outside the granuloma using a tissue excluder function of the software.

\section{Confocal Analysis of Lymph Node Tissues}

For quantification of IF stains, a Nikon A1R confocal microscope coupled to a 32-channel spectral detector was used (Nikon Instruments, Tokyo, Japan). Reference spectra were collected for each fluorophore by imaging samples single stained with the respective probes, using a single excitation source. The reference spectra were stored in a spectral library and used for off-line unmixing of all samples imaged by the spectral unmixing algorithm in the NIS elements AR software version 4.50.00 (Nikon Instruments). For analysis of colocalization, the freeware ImageJ version 2.0.0 (NIH, Bethesda, MD; http://imagej.nih.gov/ij) was used. In short, each unmixed channel was thresholded and segmented. Next, the segmented area was measured and masked before the masked areas were transformed into 8-bit images and colocalization assessed using the colocalization plugin, which highlights colocalized points of two 8-bit images. The area of the colocalized points was measured and, in the case of triplecolocalization, masked and transformed into a new 8-bit image. The new image was subsequently analyzed for colocalization against a third channel, using the same plugin.

\section{Light Microscopy Analysis of H\&E-Stained Lymph Node Tissues}

H\&E stain and light microscopy were used to assess the different types of granulomas in M. tuberculosis-infected lymph node tissues (ie, $n=8 \mathrm{~TB}$-infected and $n=8 \mathrm{~TB} /$ HIV-co-infected lymph nodes). Specific granulomas in the lymph nodes were counted and visually graded using developmental stages 1 to 4 (Table 2). Accordingly, a total of 164 granulomas in TB-infected and 117 granulomas in TB/HIV lymph node biopsy specimens were manually counted and graded using a modified version of a previously described histopathological staging of mycobacterial granulomas. ${ }^{24}$

\section{BCG-Specific IgG Enzyme-Linked Immunosorbent Assay}

The release of mycobacteria-specific IgG antibodies in plasma samples or in vitro peripheral blood mononuclear cell (PBMC) cultures obtained from the study participants was quantified using a BCG-specific enzyme-linked immunosorbent assay. Briefly, a BCG vaccine antigen (Japan BCG Laboratories, Tokyo, Japan) was used to coat Maxisorb plates (Nunc, Roskilde, Denmark) overnight at $4^{\circ} \mathrm{C}$. The plates were washed with $0.05 \%$ Tween-20 (SigmaAldrich) and blocked with $10 \%$ fetal calf serum in phosphate-buffered saline. Diluted plasma samples or PBMC culture supernatants (from PBMCs cultured without exogenous stimulation for 48 hours) from patients and controls were added (100 $\mu \mathrm{L}$ per well) and incubated for 2 hours at $37^{\circ} \mathrm{C}$ before washing and adding a rabbit antihuman IgG horseradish peroxidase conjugate (Jackson Immunoresearch Laboratories, West Grove, PA) for 2 hours at room temperature. Dilution buffer was used as a negative 
control. The enzyme-substrate reaction was developed after 20 minutes using an O-phenylenediamine (Sigma-Aldrich) substrate solution. BCG-specific IgG titers were expressed as $492 \mathrm{OD}$ and multiplied by the dilution factor.

\section{Multiplex Luminex Assay}

Inflammatory mediators [IL-1 receptor antagonist (IL-1RA), CXCL9, and CXCL10] in plasma samples were assessed using a human standard cytokine 25-plex panel (LHC0009) from Invitrogen according to the manufacturer's instructions using the Bio-Plex Luminex detection system and the BioPlex manager 4.0 curve fitting software (Bio-Rad Laboratories, Hercules, CA).

\section{mRNA Extraction and Quantitative Real-Time PCR}

PBMCs or lymph node tissue were saved in RNAlater until mRNA was extracted using the Ambion RiboPure extraction kit (Life Technologies, Carlsbad, CA) according to the manufacturer's instructions. RNA was reverse transcribed to cDNA using superscript reverse transcriptase (SuperScript VILO cDNA master mix, Invitrogen). Amplification of target genes in the cDNA samples was performed using the ABI PRISM 7700 Sequence Detection System, including the 7500 software version 2.0.6 (Applied Biosystems, Foster City, CA). Commercial FAM dye-labeled Taq-Man MGB probes and primers for Tim-3, Tbet, and GATA3 were from Sigma-Aldrich, whereas the other primers and probes were from Applied Biosystems. Cycle threshold values for the target genes were normalized to the $\mathrm{C}_{\mathrm{T}}$ value for the housekeeping gene $18 \mathrm{~S}$, and the results were analyzed by using the relative standard method as previously described. ${ }^{22,25}$ Data were presented as mRNA fold change in TB-infected or TB/HIV-co-infected samples compared with uninfected control samples $(n=5$, matched PBMC and lymph nodes tissue samples).

\section{Flow Cytometry}

Frozen PBMC samples were thawed and washed with FACS buffer [PBS containing $0.5 \%(\mathrm{v} / \mathrm{v})$ fetal calf serum and 0.5 $\mathrm{mmol} / \mathrm{L}$ EDTA] before cells were counted and stained. A total of $1 \times 10^{6}$ cells were stained for 30 minutes at $4^{\circ} \mathrm{C}$ with fluorochrome-conjugated anti-human antibodies: CD33 (phosphatidylethanolamine), CD11b (phosphatidylethanolamine-Cy7), HLA-DR (phosphatidylethanolamine-Cy5), CD15 (fluorescein isothiocyanate), CD14 (Brilliant Violet 711), and the death cell marker (Zombie UV), whereas CD3, CD19, and CD56 were assessed together in the DUMP channel (Brilliant Violet 510). CD33 and Zombie UV were obtained from Biolegend (San Diego, CA), whereas the other antibodies were from BD Pharmingen (San Diego, CA). Unstained cell samples as well as fluorescence minus one controls were used to determine the background fluorescence and to set the appropriate gates. Cells were washed twice with FACS buffer after staining and fixed with $4 \%$ formaldehyde
(Sigma-Aldrich) at room temperature for 10 minutes. After fixation, cells were washed twice with FACS buffer, and 50,000 cells were acquired from each sample by using BD LSR Fortessa (BD Biosciences) and analyzed with FlowJo version 9 (BD, Ashland, OR).

\section{Statistical Analysis}

Data are presented as medians \pm interquartile ranges or medians \pm ranges. Nonparametric analyses were used to calculate indicated $P$ values and included a Kruskal-Wallis test and Dunn posttest (when comparing more than two groups) or a MannWhitney $U$-test (when comparing two unmatched groups) or a Wilcoxon signed-rank test (when comparing two matched groups). The Spearman correlation test was used for the correlation analyses. A value of $r=1$ indicates a perfect positive correlation, whereas $r=-1$ indicates a perfect negative correlation. Statistical analyses were performed in GraphPad Prism software version 5 (GraphPad Software, San Diego, CA).

\section{Results}

\section{Enhanced Systemic Inflammation and Elevated}

Proportion of Poorly Formed and Necrotic Granulomas in Lymph Node Tissue from TB/HIV-Co-Infected Patients

Clinical and laboratory features of HIV-negative and HIVpositive patients with lymph node TB and controls are summarized in Table 1. As expected, TB/HIV-co-infected patients had significantly lower $\mathrm{CD}^{+}$T-cell counts $(P=0.001)$ and relatively higher $\mathrm{CD} 8^{+} \mathrm{T}$-cell counts in peripheral blood, resulting in a lower $\mathrm{CD} 4: \mathrm{CD} 8$ ratio $(P=0.0003)$ compared with HIV-negative patients with TB. Furthermore, hemoglobin levels were lower $(P=0.02)$ and white blood cell counts $(P=0.05)$ and erythrocyte sedimentation rates $(P=0.01)$ were higher in HIV-positive compared with HIV-negative patients with TB. Plasma HIV viral load was higher in TB/HIV-co-infected compared with HIV-positive controls $(P=0.02)$, which may suggest that viral replication is less controlled in TB/HIV co-infection.

Elevated mycobacteria-specific $\mathrm{IgG}$ production from PBMCs cultured in vitro was observed in both TB-infected $(P=0.02)$ and TB/HIV-co-infected $(P=0.0006)$ patients compared with the controls (Figure 1A). TB/HIV-co-infected patients also had enhanced mycobacteria-specific IgG responses in plasma $(P=0.0031)$ as well as elevated total IgG mRNA in lymph node tissue $(P=0.002)$ compared with HIVnegative patients with TB or controls, respectively. Multiplex profiling of plasma samples revealed an altered systemic cytokine/chemokine milieu in TB/HIV-co-infected patients, characterized by significantly elevated levels of the antiinflammatory IL-1RA $(P=0.0038)$, and increased levels of the IFN- $\gamma$-stimulated chemokines CXCL9 $(P=0.004)$ and CXCL10 $(P=0.041)$ compared with HIV-negative patients with TB as well as the controls (Supplemental Figure S1, A-C). Accordingly, there was a significant inverse correlation 

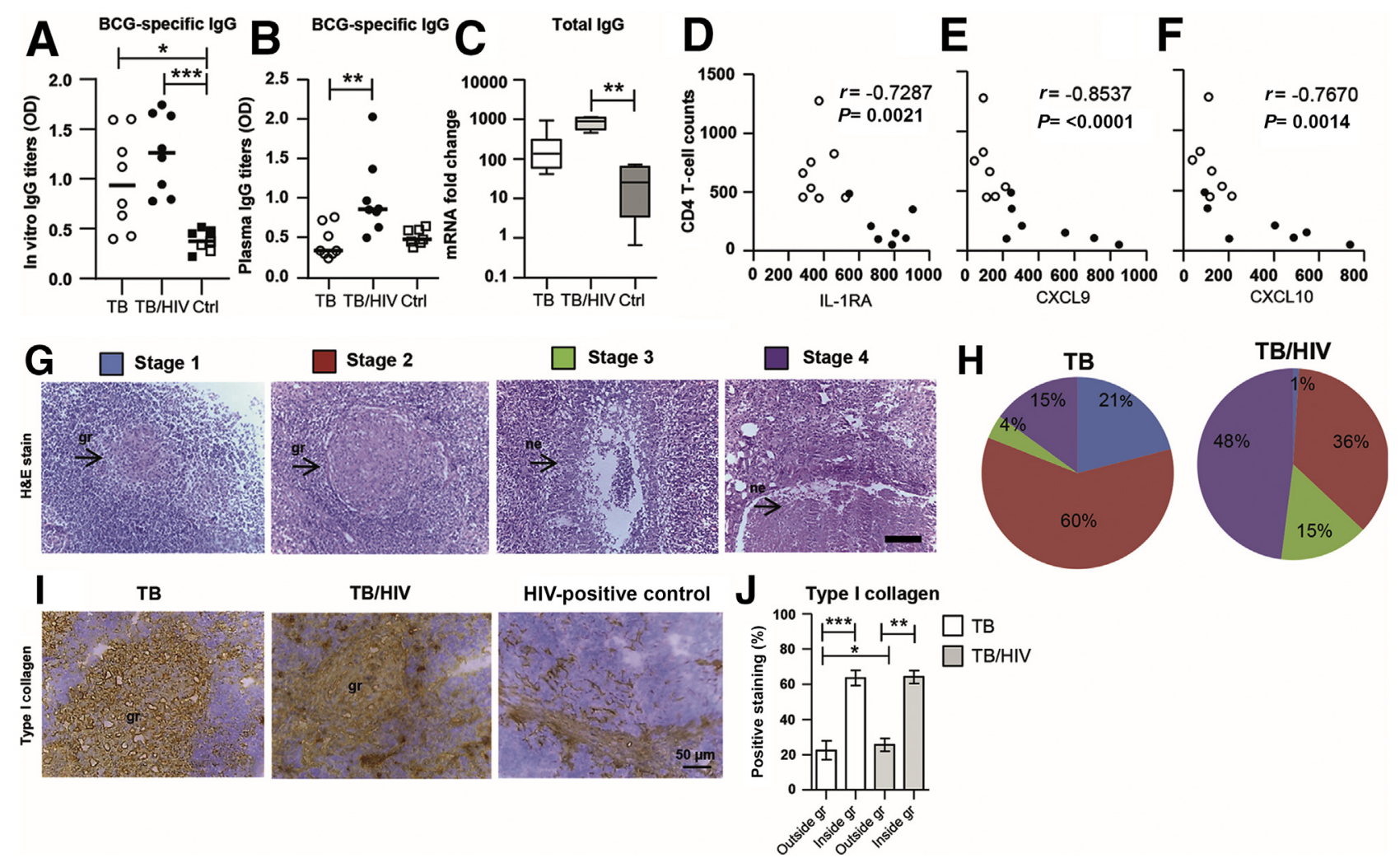

Figure 1 Characterization of systemic inflammation and tissue pathology in tuberculosis (TB)-infected and TB/HIV-co-infected patients. A-C: BCGspecific and total IgG were assessed using enzyme-linked immunosorbent assay of peripheral blood mononuclear cell (PBMC) culture supernatants (A) and serum samples (B), respectively, as well as real-time PCR of freshly isolated PBMCs (C) from TB-infected patients, TB/HIV-co-infected patients, and controls (Ctrls). D-F: Peripheral CD4 T-cell counts were inversely correlated to inflammatory mediators in plasma, including IL-1 receptor antagonist (IL-1RA) (D), CXCL9 (E), and CXCL10 (F) as assessed using the Spearman correlation test. $\mathbf{G}$ and $\mathbf{H}$ : Hematoxylin and eosin (H\&E) stain of lymph node tissue sections from a TB/HIV-co-infected patient where the arrows indicate solid or necrotic TB granulomas (G) that were manually counted and graded as stage 1 to 4 granulomas in TB-infected and TB/HIV-co-infected tissues (H). I and J: Type I collagen staining in TB-infected, TB/HIV-co-infected, and HIV-positive Ctrl tissue (I) was used to determine the level of fibrosis in TB- and TB/HIV-co-infected lymph nodes (J). $\mathbf{G}$ and I: Representative images of H\&E as well as type I collagen staining are shown. Data are expressed as medians \pm ranges or interquartile ranges. $n=8$ TB-infected patients (open circles and white bars); $n=8$ TB/ HIV-co-infected patients (closed circles and light gray bars); $n=3$ HIV-negative Ctrls (open squares); $n=4$ HIV-positive Ctrls (closed squares). ${ }^{*} P<0.05$, $* * P<0.005$, and ${ }^{* * *} P<0.001$ (Kruskal-Wallis and Dunn multiple comparisons tests or Mann-Whitney $U$-test or Wilcoxon signed-rank test). Scale bar $=50$ $\mu \mathrm{m}$. Original magnification, $\times 25$. gr, granuloma; ne, necrosis.

between $\mathrm{CD}^{+}$T-cell counts and IL-1RA $(P=0.002)$, CXCL9 $(P<0.0001)$, and CXCL10 $(P=0.0014)$ (Figure 1 , $\mathrm{D}-\mathrm{F})$, supportive of dysregulated immune activation in $\mathrm{TB} /$ HIV-co-infected patients.

Next, microscopy and computerized image analysis were applied to assess the morphology of a total of 281 TB granulomas (164 TB infected and 117 TB/HIV co-infected) in $M$. tuberculosis-infected lymph node tissues. Four major types of granulomas were observed and presented as stages 1 to 4 , with and without necrosis (Table 2 and Figure 1G). Overall, $81 \%$ of the TB granulomas in HIV-negative lymph nodes had well-defined borders and little necrosis (stage 1 to 2), whereas disrupted cellular aggregates with extensive necrosis (stage 3 to 4) were more common in TB/HIV co-infection and observed in 63\% of the granulomas (Figure 1H). Quantification of tissue fibrosis using type I collagen showed that both HIV-negative TB-infected and TB/HIV-co-infected lymph nodes contained enhanced fibrosis compared with control tissues (Figure 1I). Fibrosis was particularly high in the granulomatous areas (Figure 1I), and image analyses confirmed that granulomas in both groups demonstrated significantly elevated levels of type I collagen $(P=0.0006$ and $P=0.008$, respectively) (Figure $1 \mathrm{~J}$ ) compared with the surrounding tissue. Type I collagen expression in tissue outside the granulomas was also significantly higher $(P=0.02)$ in TB/HIV co-infection compared with TB infection (Figure 1I). Altogether, these results suggested that persistent immune activation and chronic inflammation, including poorly formed and necrotic lymph node granulomas, were more common in TB/HIV-co-infected patients compared with HIV-negative patients with TB.

\section{Elevated Expression of $M$. tuberculosis Antigen in $M$. tuberculosis-Infected Tissues}

To quantify local bacterial burden as well as viral load in the study participants, M. tuberculosis and HIV-1 antigen expression was assessed in the lymph node tissues. M. tuberculosis antigen was significantly higher in both TB-infected $(P=0.05)$ and TB/HIV-co-infected 


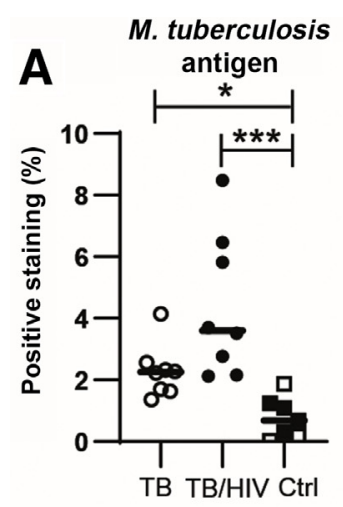

E
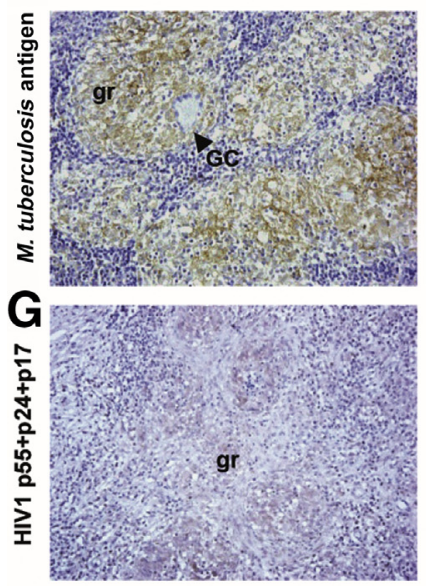

B HIV1 p55+p24+p17

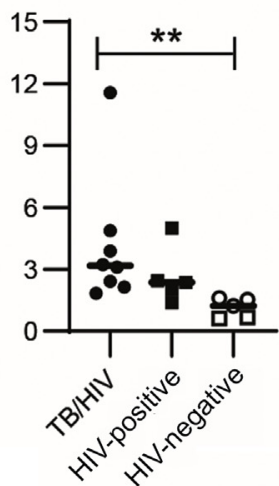

IB/HIV
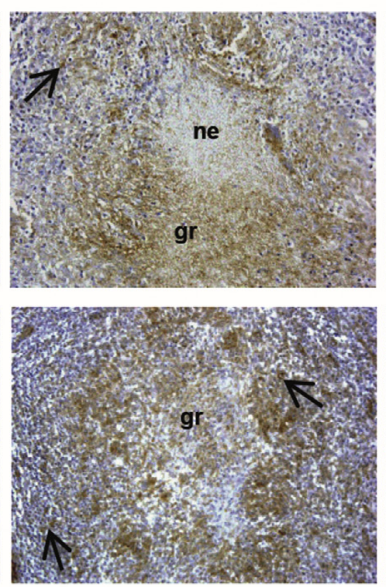

TB/HIV-co-infected patients

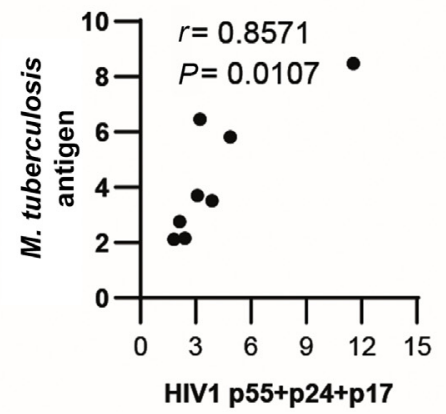

HIV-positive control

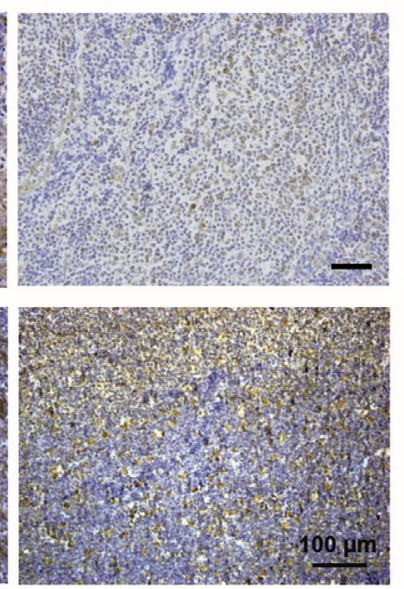

D TB/HIV-co-infected patients
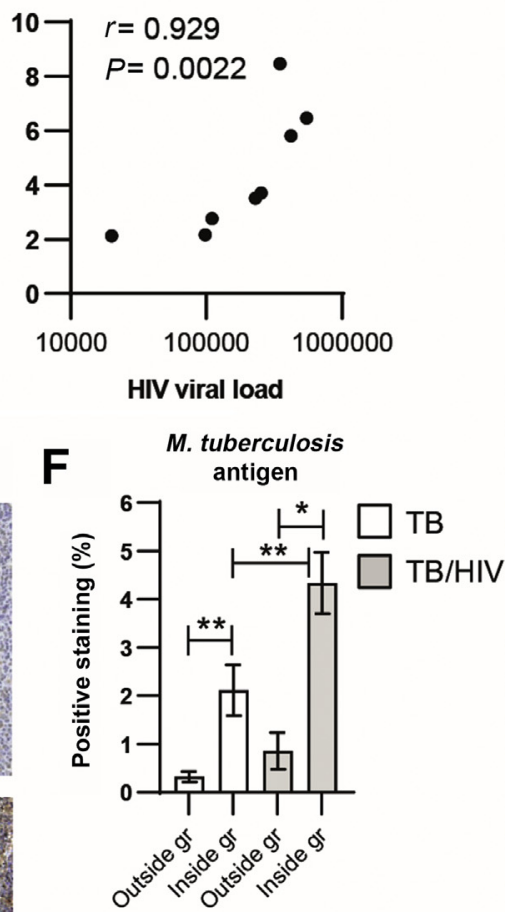

Figure 2 In situ quantification of Mycobacterium tuberculosis and HIV1 antigen load in tuberculosis (TB)-infected and TB/HIV-co-infected lymph node tissues. A and B: Immunohistochemistry and computerized image analyses was used to determine the expression (percentage of positive staining in the total cell area) and distribution of $M$. tuberculosis antigen (A) and HIV1 p55+p24+p17 (B). Note that HIV antigen stain in TB/HIV co-infection is compared with HIV-positive and HIV-negative controls (Ctrls) (3 TB-infected patients and 2 HIV-negative Ctrls). C and D: M. tuberculosis antigen expression in TB/HIV-coinfected tissue correlated to HIV antigen load in tissue (C) and to HIV viral load in plasma (D) as assessed using the Spearman correlation test. $\mathbf{E}$ and $\mathbf{F}$ : Representative images of M. tuberculosis-positive staining in TB-infected, TB/HIV-co-infected, and HIV-positive Ctrl tissue (E) and image analyses of M. tuberculosis antigen expression outside and inside the granulomatous areas in TB-infected and TB/HIV-co-infected patients is shown (F). G: Representative images of HIV1 p55+p24+p17-positive staining in TB-infected, TB/HIV-co-infected, and HIV-positive Ctrl tissue. E and G: Arrows in the images indicate positively stained cells, and arrowheads indicate giant cells (GCs). Data are expressed as medians \pm ranges or interquartile ranges. $n=8$ TB-infected patients (open circles and white bars); $n=8 \mathrm{~TB} / \mathrm{HIV}-$ co-infected patients (closed circles and light gray bars) (A, B and F); $n=3$ HIV-negative Ctrls (open squares) $; n=4$ HIV-positive Ctrls (closed squares). ${ }^{*} P<0.05,{ }^{* *} P<0.005$, and ${ }^{* * *} P<0.001$ (Kruskal-Wallis and Dunn multiple comparisons tests or Mann-Whitney $U$-test or Wilcoxon signed-rank test). Scale bar $=100 \mu \mathrm{m}$. Original magnification, $\times 16$. gr, granuloma; ne, necrosis.

$(P=0.0004)$ tissues compared with the controls, whereas $M$. tuberculosis antigen load was relatively higher in TB/ HIV-co-infected compared with HIV-negative TB-infected tissues (Figure 2A). In situ quantification of the HIV-1 antigens $\mathrm{p} 55+\mathrm{p} 24+\mathrm{p} 17$ showed a significantly $(P=0.006)$ higher expression in TB/HIV-co-infected compared with HIV-negative tissues (Figure 2B). In TB/HIV-co-infected patients, $M$. tuberculosis antigen expression in tissue correlated with HIV-1 antigen load in tissue $(r=0.86$, $P=0.0107)$ as well as HIV viral load in plasma $(r=0.93$, $P=0.0022$ ) (Figure 2, C and D). Microscopy and image analysis revealed that $M$. tuberculosis antigen expression in both TB-infected and TB/HIV-co-infected lymph nodes was significantly higher in the granulomatous areas compared with the surrounding tissue $(P=0.0078$ and $P=0.016$, respectively) (Figure 2E and F). M. tuberculosis antigen was also significantly higher in TB/HIV-co-infected granulomas compared with HIV-negative TB granulomas $(P=0.0095)$ (Figure 2F). Tissue expression of HIV-1 antigens was detected in TB/HIV granulomas but also in the lymphoid areas of both TB/HIV-co-infected and HIV-positive study participants (Figure 2G). These results support the notion that HIV can fuel M. tuberculosis replication and antigen deposition in co-infected macrophages at the site of infection. ${ }^{6}$

\section{Altered Cellular Composition in TB/HIV-Co-Infected Lymph Nodes}

IHC (Supplemental Figure S2, A-D) and image analyses revealed a significant reduction $(P=0.002)$ of $\mathrm{CD}^{+} \mathrm{T}$ 

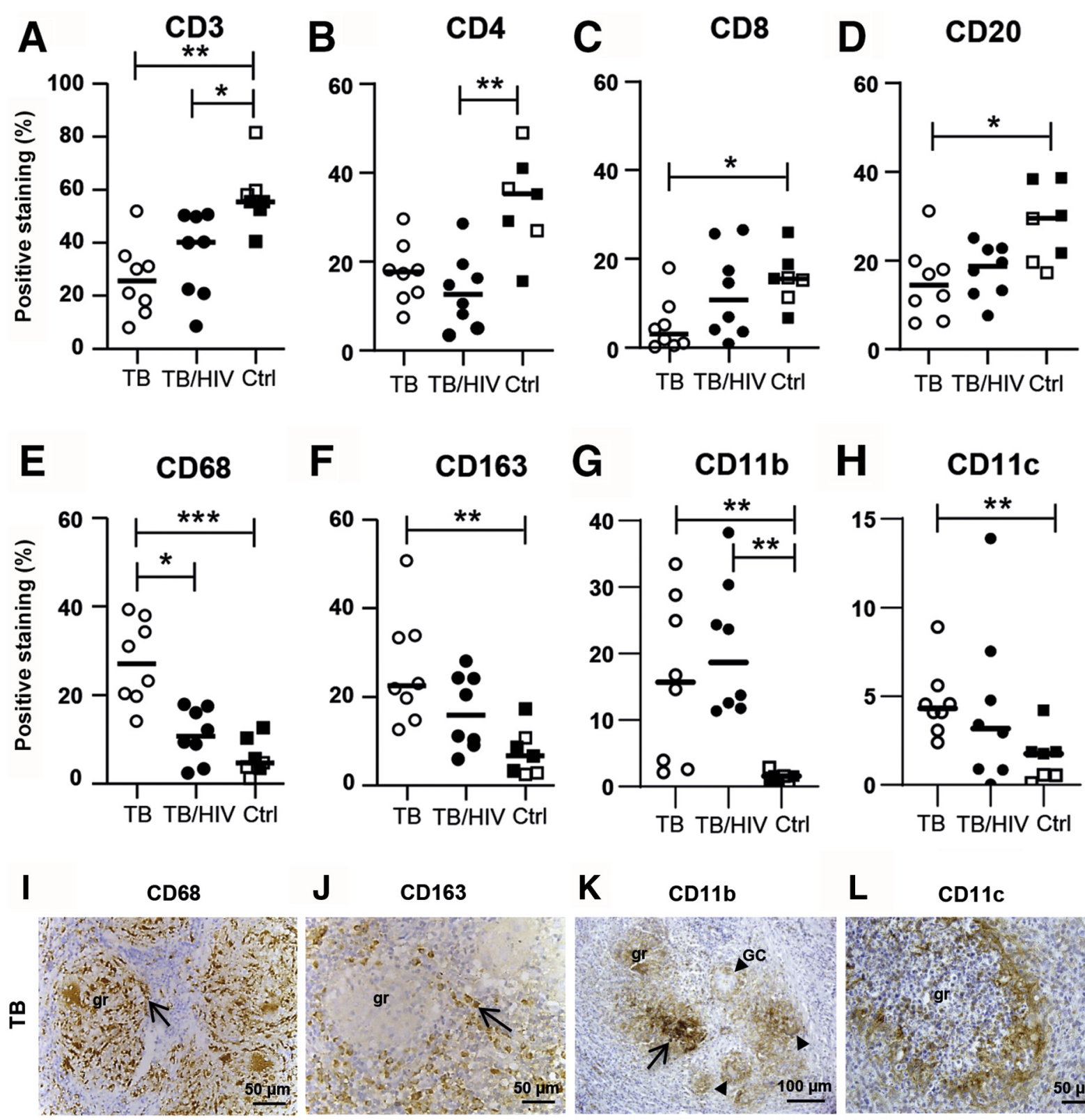

J CD163
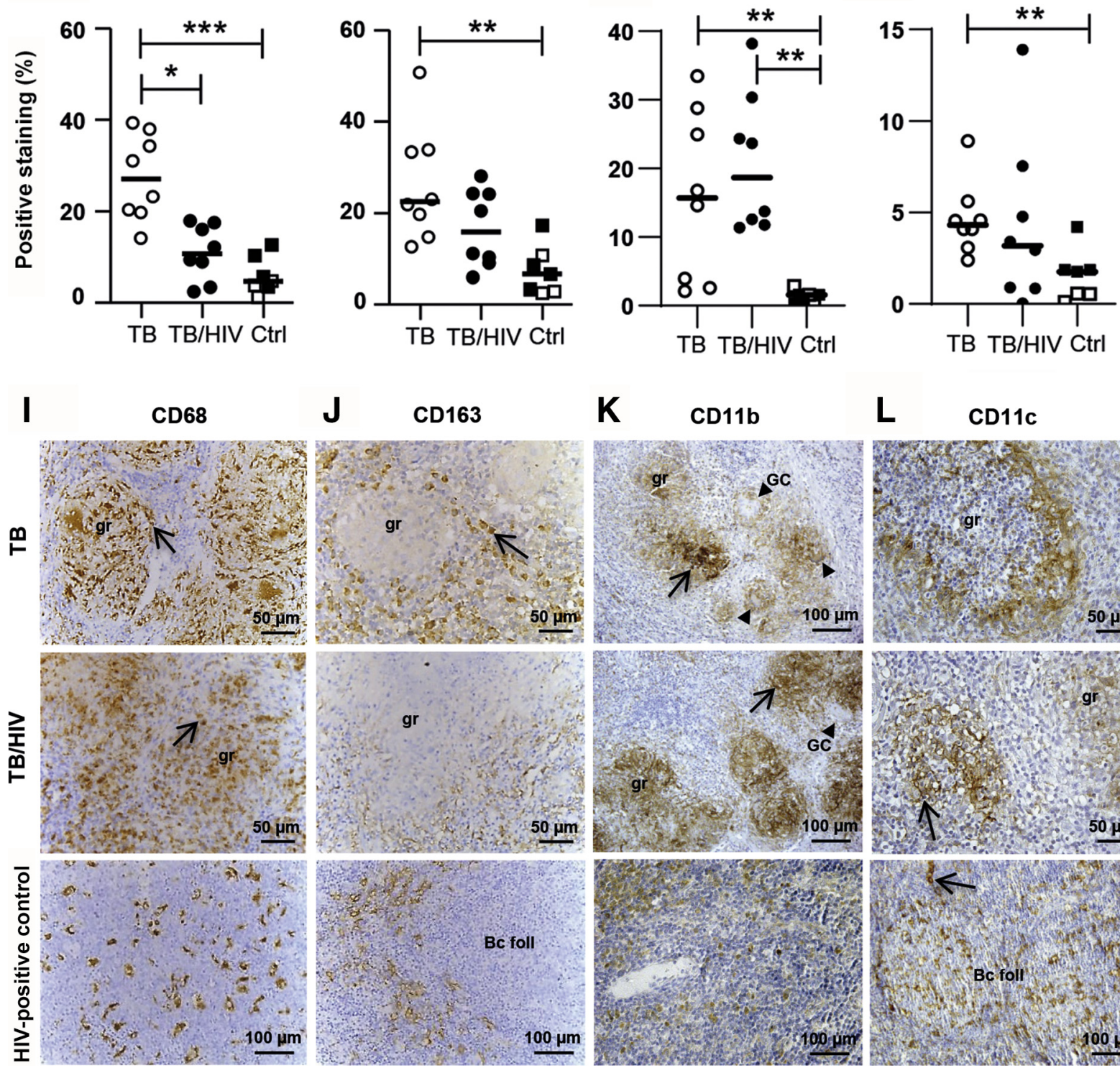

L CD11c
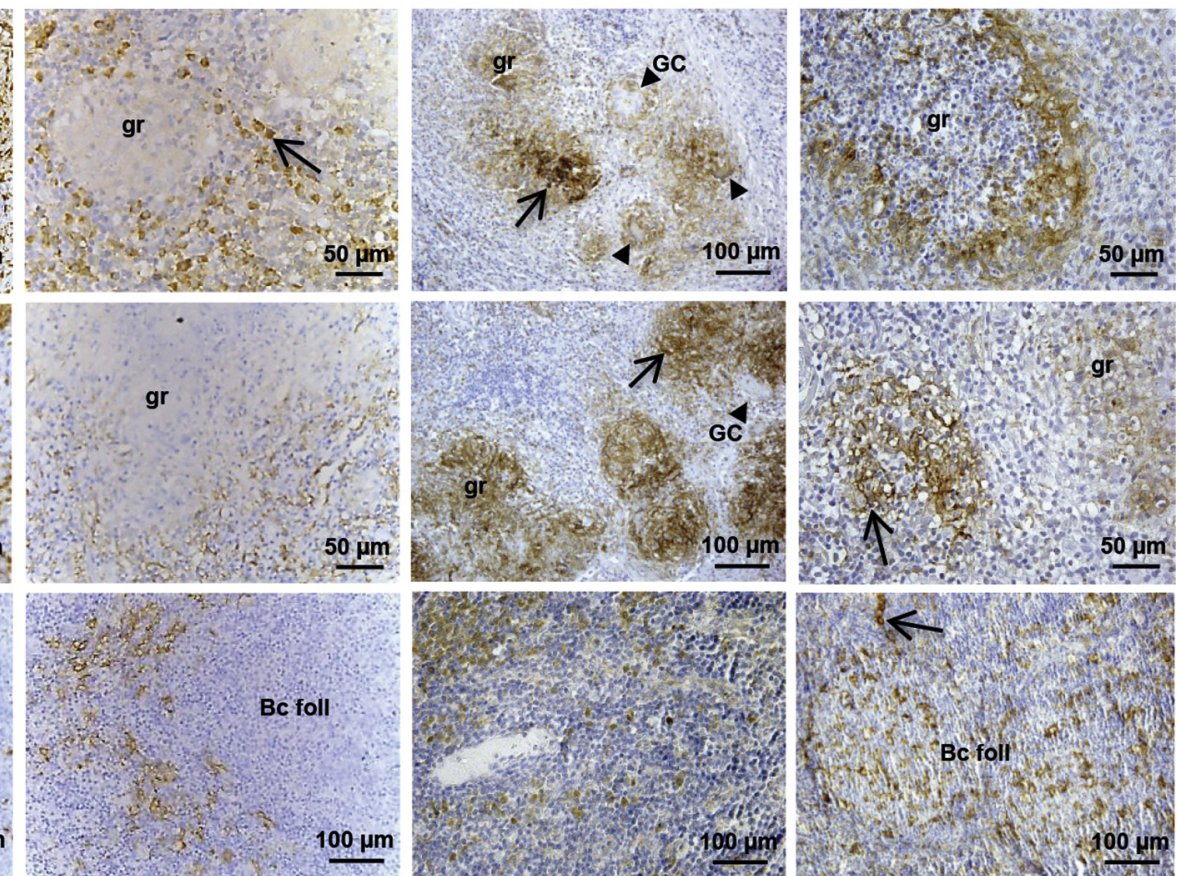

Bc foll

$100 \mu \mathrm{m}$

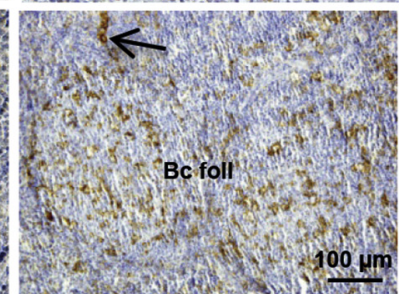


cells in both TB-infected and TB/HIV-co-infected lymph nodes (Figure $3 \mathrm{~A}$ ). Although $\mathrm{CD} 4^{+} \mathrm{T}$ cells were significantly reduced in TB/HIV co-infection $(P=0.007)$ (Figure $3 \mathrm{~B}$ ), $\mathrm{CD}^{+} \mathrm{T}$ cells were significantly reduced in HIV-negative TB tissues $(P=0.04)$ (Figure 3C). TBinfected $(P=0.03)$ and TB/HIV-co-infected patients had reduced levels of $\mathrm{CD} 20^{+} \mathrm{B}$ cells in lymph node tissue compared with the controls (Figure 3D), and these reduced levels were particularly pronounced in HIV-negative TBinfected tissues.

In situ expression and distribution of different myeloid markers showed a significant increase of $\mathrm{CD}^{+} 8^{+}$ $(P=0.0007)$ and $\mathrm{CD} 163^{+}(P=0.005)$ macrophages as well as $\mathrm{CD}_{11 b^{+}}(P=0.0097)$ and $\mathrm{CD} 11 \mathrm{c}^{+}(P=0.031)$ cells in HIV-negative TB-infected lymph nodes tissues compared with controls (Figure 3, E-H). $\mathrm{CD} 68^{+}$macrophages were also higher in TB-infected compared with TB/ HIV-co-infected tissues $(P=0.001)$ (Figure 3E). The only myeloid marker that was up-regulated in TB/HIV-coinfected tissues compared with the control was CD11b $(P=0.0022)$, whereas CD68, CD163, and CD11c were all relatively lower compared with HIV-negative TB tissues (Figure 3, E-H). $\mathrm{CD}^{+} 8^{+}$granulomas with well-defined borders were less frequent in TB/HIV-co-infected lymph nodes (Figure 3I) and $\mathrm{CD}^{+} 8^{+}$cells were confined inside the M. tuberculosis granulomas, whereas $\mathrm{CD} 163^{+}$cells were located at the periphery, surrounding $\mathrm{CD}^{+} 8^{+}$macrophage clusters (Figure 3, I and J). Most CD11 $\mathrm{b}^{+}$and $\mathrm{CD} 11 \mathrm{c}^{+}$cells were located in the granulomatous lesions of TB-infected and TB/HIV-co-infected tissues, but some were also distributed in the lymphoid areas of the lymph nodes (Figure 3, K and L). The size and morphology of CD11 $\mathrm{b}^{+}$ cells were usually smaller and more rounded compared with $\mathrm{CD} 11 \mathrm{c}^{+}$cells, which were larger and more DC-like in the patient as well as control tissues (Figure 3, K and L). Overall, altered cellular dynamics in TB-infected and TB/ HIV-co-infected lymph nodes involved extensive tissue remodeling, including reduced expression of both $\mathrm{CD} 4^{+}$ and $\mathrm{CD} 8^{+} \mathrm{T}$ cells and $\mathrm{CD} 20^{+} \mathrm{B}$ cells but elevated myeloid cell subsets. A lower expression of CD68 and the M2associated CD163 receptor but elevated levels of the cell surface integrin CD11b in TB/HIV-co-infected tissues may suggest the presence of an aberrant myeloid cell population in these patients.

\section{mRNA Expression Profiling Suggests Elevated} Inflammation and Increased Immune Inhibition at the Site of Infection in TB/HIV-Co-Infected Patients

Figure 4A illustrates mRNA expression profiling of lymph node tissue and peripheral blood cells from TBinfected compared with TB/HIV-co-infected patients. Overall, the data demonstrate that the transcriptional landscape at the site of TB infection may be relatively different compared with peripheral blood. Compared with HIV-negative TB-infected patients, PBMCs from TB/ HIV-co-infected patients displayed a relative increase of CD8, IL-6, total IgG, Arg-1, and lymphocyte activated gene (LAG)-3 mRNA. In the lymph nodes, there was an increased mRNA expression of IL- 6 , IFN- $\gamma$, IL-21, Arg1, total IgG, IDO, and forkhead box P3 (FoxP3) in both TB-infected and TB/HIV-co-infected tissues compared with the uninfected controls.

mRNA expression of selected molecules in TB-infected lymph nodes tissues compared with controls is demonstrated in Figure 4, B-M. TB/HIV-co-infected tissues expressed significantly more T-bet $(P=0.029), \mathrm{IFN}-\gamma$ $(P=0.05)$, and IL-10 $(P=0.022)$ mRNA compared with TB (Figure 4, B, E and G), whereas the HIV-negative TB group displayed higher levels of Th2 markers, such as GATA-binding protein $3(P=0.016)$ and IL-13 $(P=0.014)$ mRNA (Figure $4, \mathrm{C}$ and $\mathrm{F})$. This finding is consistent with an up-regulation of the inflammatory chemokines CXCL9 and CXCL10 in TB/HIV co-infection (Supplemental Figure S1, B and C) and an enhanced tissue expression of the M2-marker CD163 in HIV-negative TB-infected patients (Figure 3, F and J). Moreover, FoxP3 expression was up-regulated in both TB-infected $(P=0.009)$ and TB/HIV-co-infected $(P=0.044)$ patients compared with the controls (Figure 4D). Likewise, the pleiotropic inhibitors suppressor of cytokine signaling (SOCS)-3, LAG-3, and T-cell immunoglobulin mucin (TIM)-3 were all up-regulated in TB/HIV co-infection compared with the controls $(P=0.05, P=0.02$, and $P=0.009$, respectively), and LAG-3 and TIM-3 were also significantly higher in TB/HIV co-infection compared with TB infection $(P=0.005$ and $P=0.009)$ (Figure $4, \mathrm{H}-\mathrm{J})$.

Several T-cell-specific effector molecules as well as the innate antimicrobial peptide human cationic antibacterial

Figure 3 Immune cell composition and morphology in tuberculosis (TB)-infected and TB/HIV-co-infected lymph node tissues. A-H: Immunohistochemistry and computerized image analyses was used to determine the expression (percentage of positive staining in the total cell area) and distribution of lymphocytes (A-D) and myeloid cells (E-H): CD3 (A), CD4 (B), and CD8 T cells (C), CD20 B cells (D), as well as CD68 (E), CD163 (F), CD11b (G), and CD11C (H) myeloid subsets. I-L: Representative images of CD68-positive (I), CD163-positive (J), CD11b-positive (K), and CD11C-positive (L) staining in TB-infected, TB/HIV-co-infected, and HIV-positive control (Ctrl) tissue. Arrows in the images indicate positively stained cells, and arrowheads indicate giant cells (GCS). Data are expressed as medians \pm ranges; $n=8$ TB-infected patients (open circles); $n=8 \mathrm{~TB} / \mathrm{HIV}-$ co-infected patients (closed circles); $n=3$ HIV-negative Ctrls (open squares); $n=4$ HIV-positive Ctrls (closed squares). ${ }^{*} P<0.05,{ }^{* *} P<0.005$, and ${ }^{* *}{ }^{*} P<0.001$ (Kruskal-Wallis and Dunn multiple comparisons tests). Original magnification: $\times 16$ (I, top and middle rows; J, top and middle rows; L, top and middle rows); $\times 25$. Scale bars, $50 \mu \mathrm{m}$ ( $\mathrm{I}$, top and middle rows; J, top and middle rows; L, top and middle rows); $100 \mu \mathrm{m}$ (I, bottom row; J, bottom row; K; L, bottom row). BC foll, B-cell follicle; gr, granuloma. 

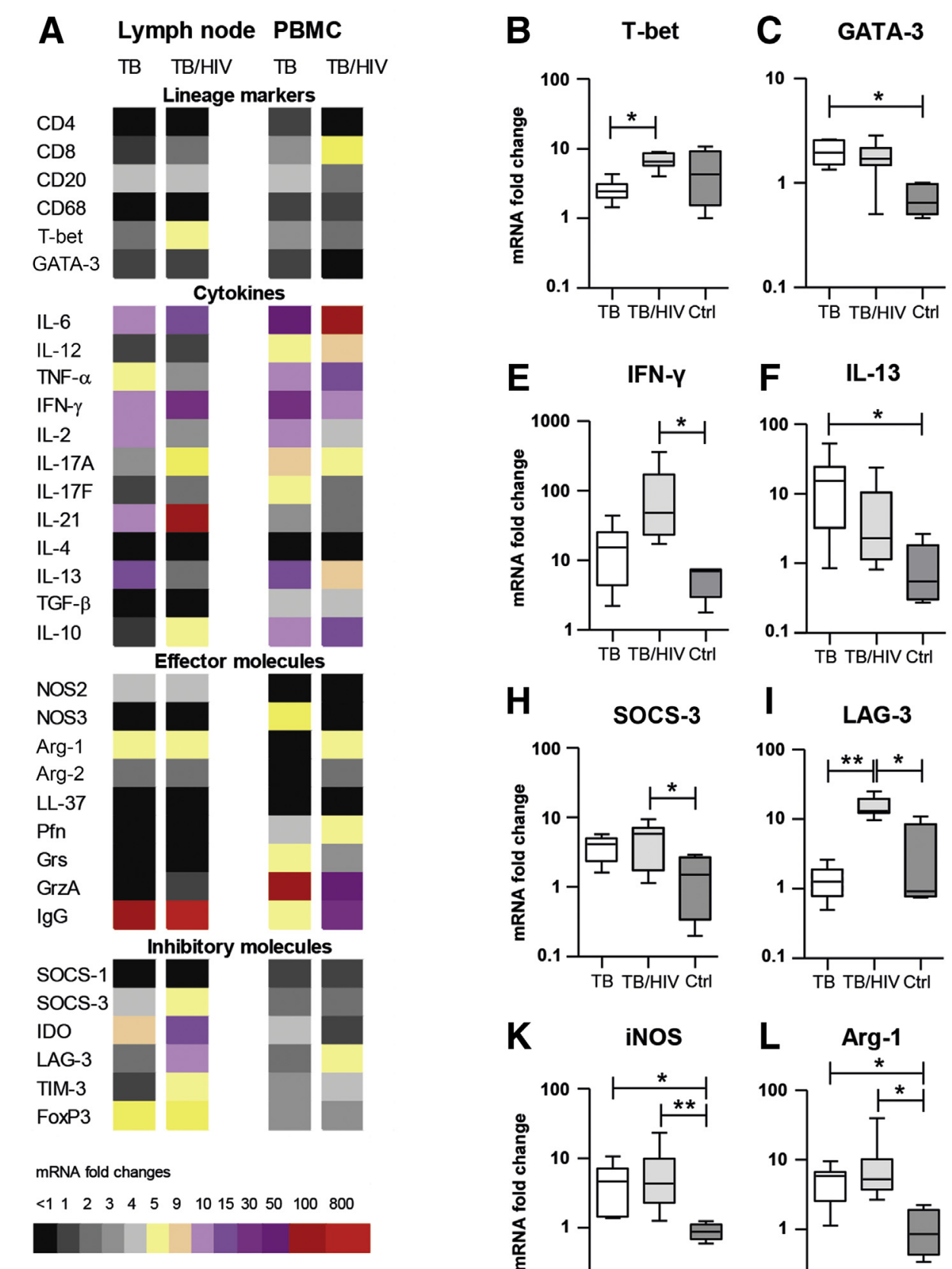

D FoxP3
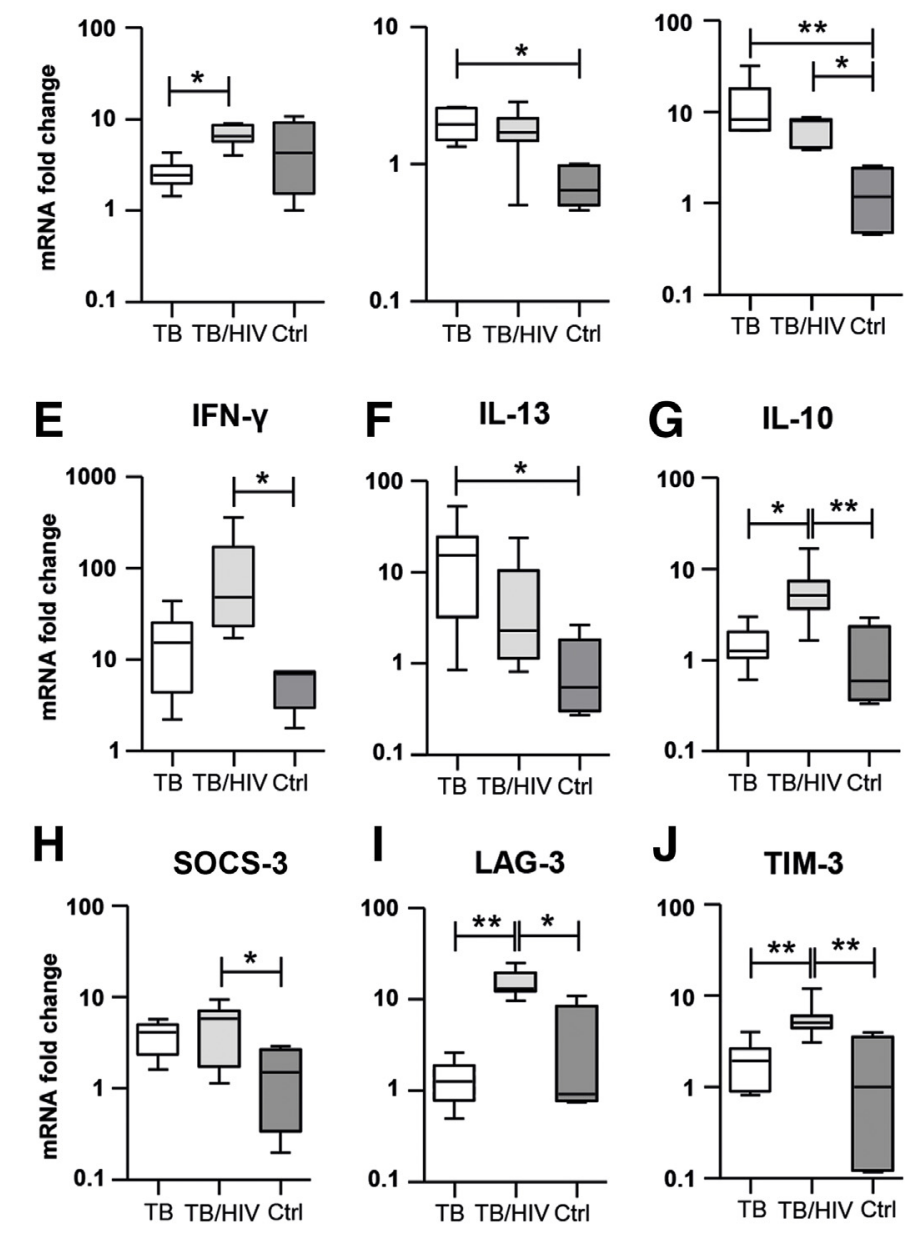

G IL-10
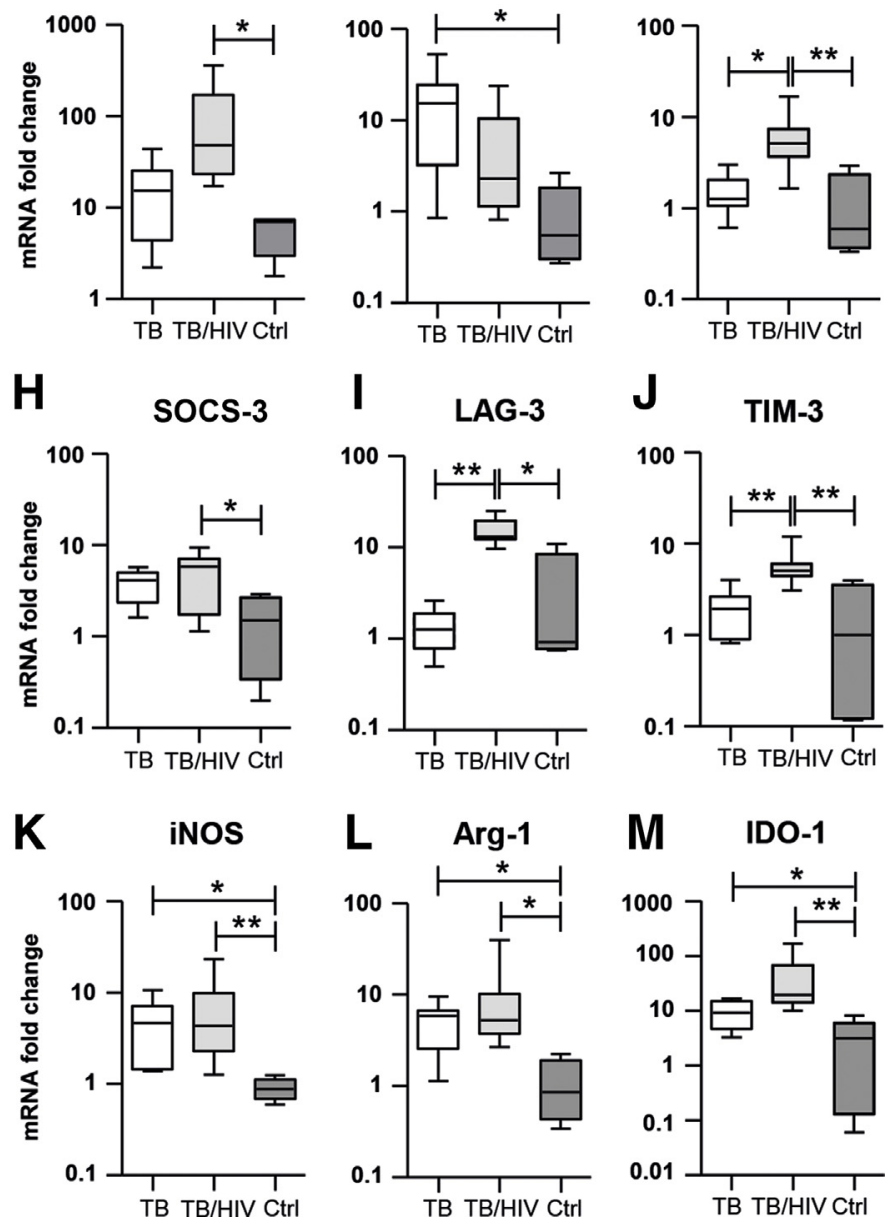

Figure 4 RNA profiling of lymph node tissues and peripheral blood mononuclear cells (PBMCs) from tuberculosis (TB)-infected and TB/HIV-co-infected patients. A: Heatmap demonstrating mRNA expression (fold change) of a number of immune mediators as determined by quantitative real-time PCR. B-M: $m R N A$ expression of selected immune molecules in lymph node tissues. Data are expressed as medians \pm ranges. $n=8$ TB-infected patients (white bars); $n=8$ TB/HIV-co-infected patients (light gray bars); $n=5$ matched PBMC and lymph nodes tissue samples (dark gray bars). ${ }^{*} P<0.05, * * P<0.005$ (Kruskal-Wallis and Dunn multiple comparisons tests). Arg, arginase; (trl, control; eNOS, endothelial nitric oxide synthase; FoxP3, forkhead box P3; GATA-3, GATA-binding protein 3; Grs, granulysin; GrzA, granzyme A; IDO-1, indoleamine 2,3-dioxygenase-1; IFN- $\gamma$, interferon- $\gamma$; iNOS, inducible nitric oxide synthase; LAG-3, lymphocyte activated gene-3; LL-37, human cationic antibacterial protein of $18 \mathrm{kDa}$; NOS, nitric oxide synthase; Pfn, perforin; SOCS, suppressor of cytokine signaling; TIM-3, T-cell immunoglobulin mucin3 ; TNF- $\alpha$, tumor necrosis factor- $\alpha$.

protein of $18 \mathrm{kDa}$ (LL-37) were expressed at low levels at the site of infection in TB and TB/HIV lymph node tissues (Figure 4A). Instead, the cell-signaling enzymes iNOS, Arg-1, and IDO were significantly up-regulated in both TB $(P=0.019, P=0.036$, and $P=0.019$, respectively $)$ and TB/HIV $(P=0.0072, P=0.022$, and $P=0.0012$, respectively) compared with the control group (Figure 4, $\mathrm{K}-\mathrm{M}$ ). Importantly, differences in mRNA expression of most immune molecules shown in Figure 4 were only evident at the site of infection and not in corresponding samples from peripheral blood (Supplemental Figure S3). 
Differential Distribution of iNOS, Arg-1, and IDO

Expression in M. tuberculosis-Infected Granulomatous Tissue

To further explore the phenotype and function of macrophages in the granulomatous tissue, the cell-signaling enzymes iNOS, Arg-1, and IDO were assessed. There was a significant increase of both iNOS $(P=0.003)$ and $\operatorname{Arg}-1(P=0.004)$ expression in the TB/HIV-co-infected group, although iNOS was also relatively higher in the TB-infected group compared with the controls (Figure 5, A and B). Notably, both iNOS (median of 4.64\% HIV positive versus $0.29 \%$ HIV negative) and Arg-1 (median of $0.47 \%$ HIV positive versus $0.05 \%$ HIV negative) were up-regulated in the HIV-infected controls (Figure 5, A
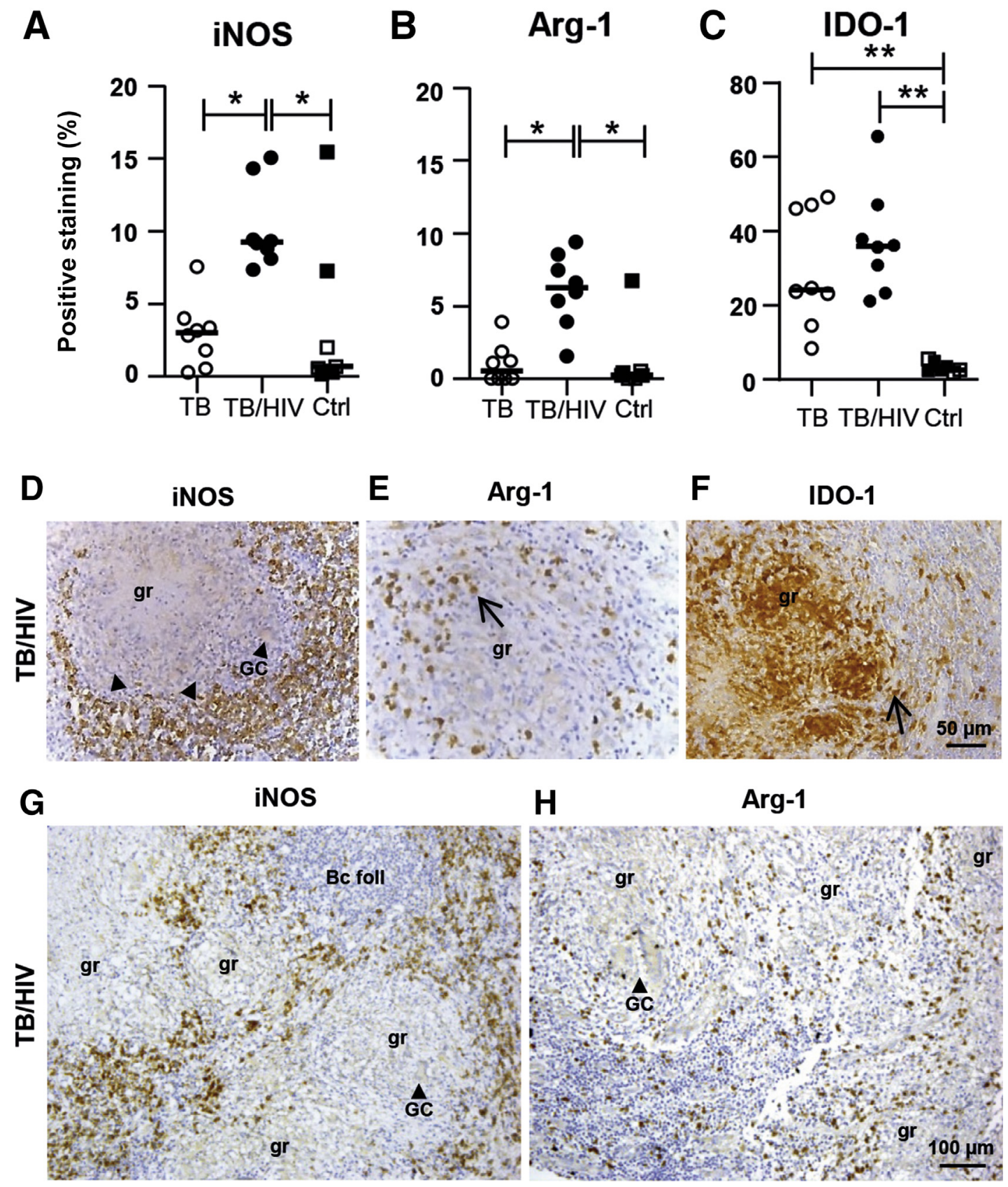

Figure 5 In situ expression of cell-signaling enzymes in tuberculosis (TB) infected-and TB/HIV-co-infected lymph node tissues. A-C: Immunohistochemistry and computerized image analyses were used to determine the expression (percentage of positive staining in the total cell area) and distribution of inducible nitric oxide synthase (iNOS) (A), arginase (Arg)-1 (B), and indoleamine 2,3-dioxygenase (IDO) (C). D-F: Representative images of iNOS (D), Arg-1 (E), and IDO (F) staining in TB/HIV-co-infected lymph node tissue. $\mathbf{G}$ and $\mathbf{H}$ : iNOS (G) and Arg-1 (H) at a lower magnification visualizing staining patterns in confluent granulomas. Arrows indicate positively stained cells, and arrowheads indicate giant cells (GCs). Data are expressed as medians \pm interquartile ranges. $n=8 \mathrm{~TB}$-infected patients (open circles); $n=8 \mathrm{~TB} / \mathrm{HIV}$-co-infected patients (closed circles); $n=3$ HIV-negative controls (Ctrls) (open squares); $n=4$ HIV-positive Ctrls (closed squares). ${ }^{*} P<0.05,{ }^{*} P<0.005$ (Kruskal-Wallis and Dunn multiple comparisons tests). Scale bars: $50 \mu \mathrm{m}$ (D-F); $100 \mu \mathrm{m}$ ( $\mathbf{G}$ and $\mathbf{H})$. Original magnification: $\times 16(\mathbf{D}-\mathbf{F}) ; \times 25$ (G and $\mathbf{H})$. Bc foll, B-cell follicle; gr, granuloma. 

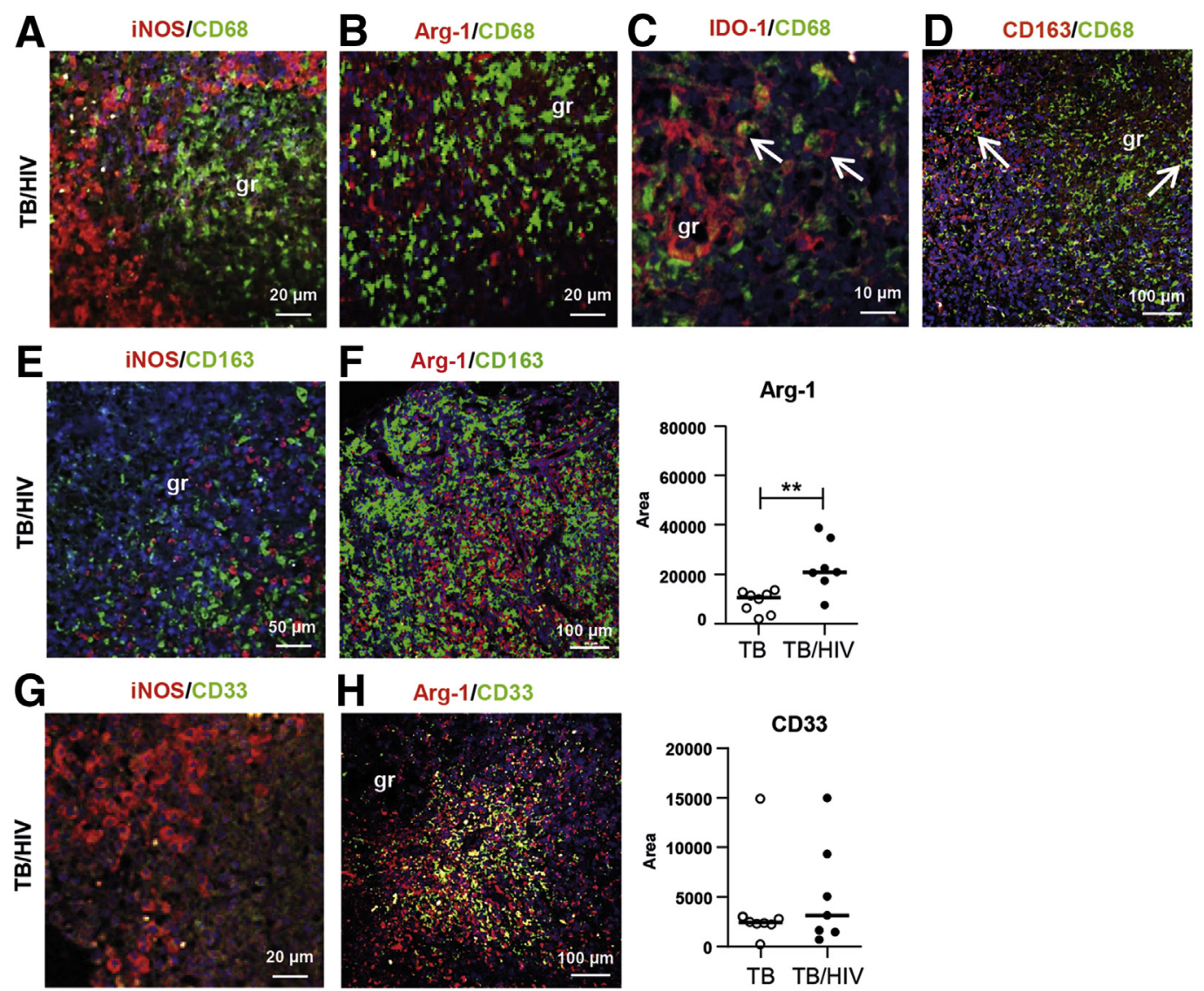

H $\quad$ Arg-1/CD 33
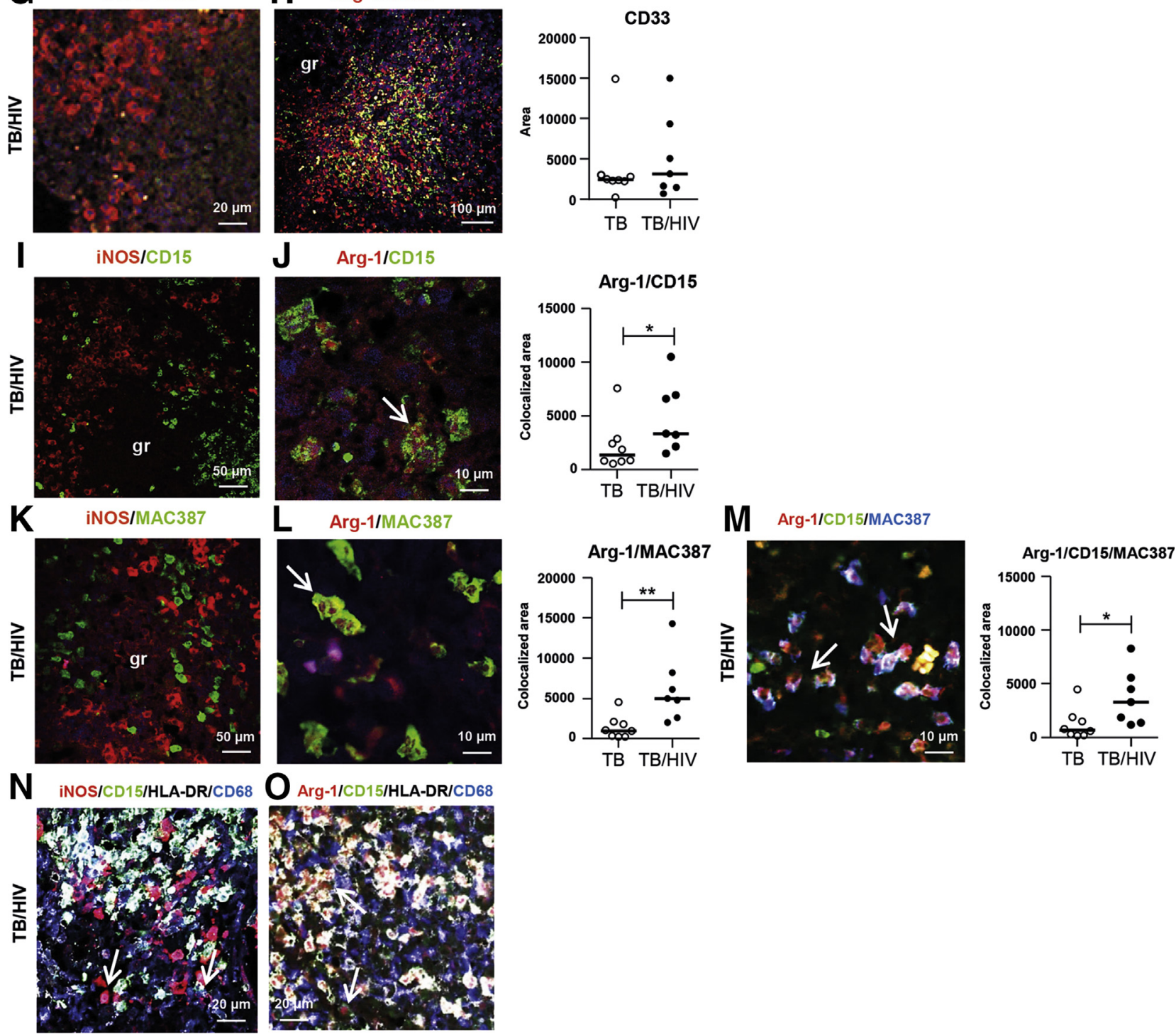
and B). Expression of the inhibitory molecule IDO was strongly enhanced $(P=0.0008)$ in both patient groups (Figure 5C). Interestingly, iNOS- and Arg-1-producing cells were mostly localized at the periphery of the TB granulomas in cells with a mononuclear morphology (Figure 5, D and E), whereas IDO-1 expression was detected primarily inside the granulomas (Figure 5F). Figure 5, G and $\mathrm{H}$ illustrates that iNOS and Arg-1 expression in and around confluent granulomas was mostly confined to the T-cell-rich areas surrounding the cores of the lesions. iNOS-expressing cells were also very few in the B-cell follicles (Figure 5G). Arg-1 expression was generally less stringent than iNOS, and numerous Arg-1-producing cells were found in the peripheral rim of the granulomatous lesions and in the lymphoid areas (Figure 5H). Multinucleated giant cells in the center of the granuloma expressed iNOS at a low intensity compared with the iNOS-positive cells surrounding the granuloma (Figure 5, D and G). Likewise, Arg-1 was expressed at lower intensity in giant cells and polymorphonuclear cells in and around the necrotic areas in the granulomas (Figure 5H and Supplemental Figure S4A).

\section{Elevated Expression of $\mathrm{Lin}^{-} / \mathrm{CD}_{3}{ }^{+} \mathrm{CD}_{15}{ }^{+} / \mathrm{MAC} 387^{+}$ $/ \mathrm{HLA}^{-D R^{i n t}} / \mathrm{Arg}-1^{+}$Cells Characteristic of MDSCs in TB/HIV-Co-Infected Tissue}

Multicolour confocal microscopy demonstrated that neither iNOS nor Arg-1 co-localized with CD68-positive or CD163positive macrophages (Figure 6, A, B, E and F). In contrast, IDO-1 expression co-localized largely with $\mathrm{CD}^{+} 8^{+}$macrophages, although IDO was also expressed in some CD68negative cells (Figure 6C). Consistent with the nonoverlapping expression of CD68 and CD163 detected with IHC (Figure 3, I and J), IF confirmed that CD68 and CD163 coexpression was low in both TB-infected (data not shown) and TB/HIV-co-infected tissues (Figure 6D). Overall, coexpression of iNOS could not be detected in $\mathrm{CD}^{+} 8^{+}$or $\mathrm{CD} 163^{+}$cells, $\mathrm{CD}^{+} \mathrm{T}$ cells, or $\mathrm{CD}^{+} 0^{+} \mathrm{B}$ cells (Supplemental Figure S4, B-E) or in subsets, including $\mathrm{CD}^{+} 6^{+}$natural killers cells or $\mathrm{CD} 123^{+}$plasmacytoid DCs (data not shown), which were very few in the TB tissue. On testing of myeloid markers, iNOS did not co-localize with $\mathrm{CD} 33^{+}, \mathrm{CD} 5^{+}$, or MAC $387^{+}$(Figure 6, G, I and K) but was expressed in CD68-negative cells with low or intermediate
HLA-DR expression (Figure 6N). Similar to iNOS, IDO was not expressed in $\mathrm{CD} 15^{+}$or MAC $387^{+}$cells (data not shown).

The confocal analyses further revealed that Arg1 -expressing cells were negative for lineage-specific markers CD3, CD20, CD56, and CD123 (data not shown). However, Arg-1 co-localized considerably with $\mathrm{CD}_{3}{ }^{+}, \mathrm{CD}_{15}{ }^{+}$, and $\mathrm{MAC} 387^{+}$cells with low or intermediate expression of HLA-DR (Figure 6, H, J, L, M, and $\mathrm{O}$ ), suggestive of an MDSC cell subset. Quantification of co-expression revealed a significantly elevated expression of $\operatorname{Arg}-1^{+}(P=0.006), \operatorname{Arg}-1^{+} \mathrm{MAC} 387^{+}(P=0.002)$, Arg$1^{+} \mathrm{CD} 15^{+}$double-positive $(P=0.05)$, and Arg$1^{+} \mathrm{CD} 15^{+} \mathrm{MAC} 387^{+}$triple-positive $(P=0.03)$ cells in TB/ HIV co-infection compared with TB infection (Figure 6, F, $\mathrm{J}, \mathrm{L}$ and $\mathrm{M})$. TB/HIV-co-infected tissues demonstrated a high co-localization of Arg-1 with $\mathrm{CD}^{+} 3^{+}$cells located primarily around the core of the TB granulomas, although there was no difference in CD33 expression in TB-infected compared with TB/HIV-co-infected tissues (Figure $6 \mathrm{H}$ ). Altogether, these results demonstrate the presence of elevated levels of cells characteristic of MDSCs in the microenvironment of lymph nodes granulomas from $\mathrm{TB} /$ HIV-co-infected patients.

\section{Elevated Levels of Granulocytic MDSCs in the Peripheral Circulation of TB/HIV-Co-Infected Patients}

To explore whether MDSCs were present in the peripheral circulation of TB-infected patients, TB/HIV-co-infected patients, and controls, flow cytometry staining was performed using corresponding PBMC samples from the study participants. In humans, MDSCs have been characterized as

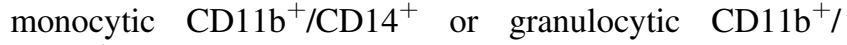
$\mathrm{CD} 15^{+}$MDSCs and are broadly defined as lymphocyte lineage-negative cells that express the common myeloid marker CD33 together with low or no expression of mature myeloid cell markers, such as HLA-DR, CD40, or CD80. ${ }^{10,19,26}$ Accordingly, a gating strategy was used for identification of monocytic $\left(\mathrm{CD} 14^{+}\right)$or granulocytic $\left(\mathrm{CD} 15^{+}\right)$MDSC subsets in peripheral blood based on lineage-negative (CD3, CD19, CD56) and HLA-DR-negative or low cells expressing the myeloid markers $\mathrm{CD} 33^{+}$ and $\mathrm{CD}_{11 \mathrm{~b}^{+}}$(Supplemental Figure S5). ${ }^{27}$ Flow cytometry showed that MDSCs identified among Lin $^{-} / \mathrm{HLA}-\mathrm{DR}^{\text {low }}$ /

\footnotetext{
Figure 6 Confocal imaging and analyses of myeloid cell markers in tuberculosis (TB)-infected and TB/HIV-co-infected lymph node tissues. A-0: Immunofluorescence and confocal microscopy analyses were used to determine the expression (colocalized area) and distribution of the following markers: inducible nitric oxide synthase (iNOS) (red)/CD68 (green) (A), arginase (Arg)-1 (red)/CD68 (green) (B), indoleamine 2,3-dioxygenase (ID0) (red)/CD68 (green) (C), CD163/CD68 (green) (red) (D), iNOS (red)/CD163 (green) (E), Arg-1 (red)/CD163 (green) (F), iNOS (red)/(CD33) (green) (G), Arg-1 (red)/(CD33) (green) (H), iNOS (red)/(CD15) (green) (I), Arg-1 (red)/(CD15) (green) (J), iNOS (red)/(MAC387) (green) (K), Arg-1 (red)/(MAC387) (green) (L), Arg-1 (red)/ (CD15) (green)/MAC387 (blue) (M), iNOS (red)/CD15 (green)/HLA-DR (white)/CD68 (blue) (N), and Arg-1 (red)/CD15 (green)/HLA-DR (white)/CD68 (blue) (0). Representative images of iNOS or Arg-1 double, triple, or quadruple stains are shown in TB/HIV-co-infected lymph node tissue. F-M: Positively stained area of Arg-1 (F) or CD33 (H) and colocalized area of Arg-1 and CD15 (J) or MAC387 (L) or Arg-1 and CD15 and MAC387 (M) was quantified using digital image analyses. Arrows indicate double-positive or triple-positive cells. Data are expressed as medians \pm ranges. $n=8$ TB-infected patients (open circles); $n=7$ TB/HIV-co-infected patients (closed circles). ${ }^{*} P<0.05,{ }^{*} P<0.005$ (Mann-Whitney $U$-test). Original magnification: $\times 63(\mathbf{A}, \mathbf{B}, \mathbf{G}, \mathbf{N}$, and $\mathbf{0}) ; \times 100(\mathbf{C}, \mathbf{J}, \mathbf{L}$, and $\mathbf{M}) ; \times 16(\mathbf{D}, \mathbf{F}$, and $\mathbf{H}) ; \times 25(\mathbf{E}, \mathbf{I}$, and $\mathbf{K})$. Scale bars: $20 \mu \mathrm{m}(\mathbf{A}, \mathbf{B}, \mathbf{G}, \mathbf{N}$, and $\mathbf{0}) ; 10 \mu \mathrm{m}(\mathbf{C}, \mathbf{J}, \mathbf{L}$, and $\mathbf{M}) ; 100 \mu \mathrm{m}(\mathbf{D}, \mathbf{F}$, and $\mathbf{H}) ; 50 \mu \mathrm{m}(\mathbf{E}, \mathbf{I}$, and $\mathbf{K})$. gr, granuloma.
} 

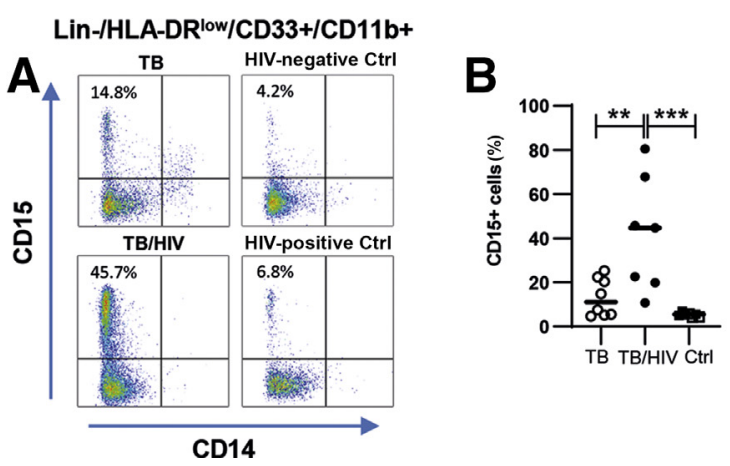

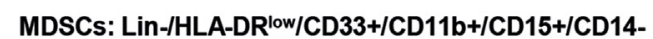
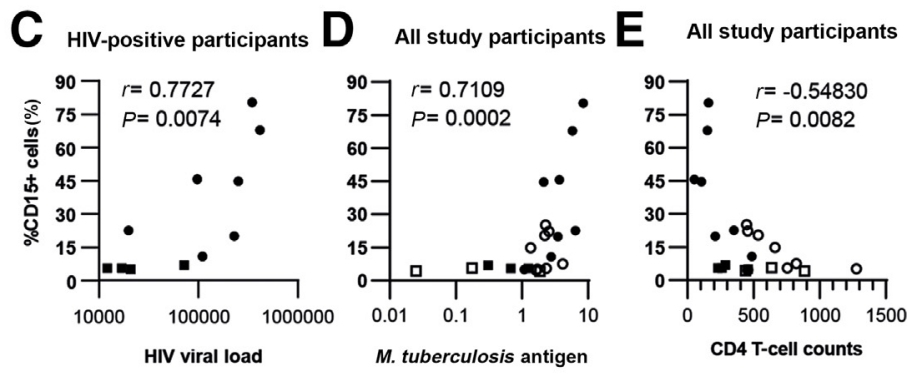

Figure 7 Flow cytometric analyses of cells characteristic of myeloid-derived suppressor cells (MDSCs) present in peripheral blood from tuberculosis (TB)infected and TB/HIV-co-infected patients. A: Representative dot plots showing MDSCs present in TB-infected or TB/HIV-co-infected patients as well as HIVnegative and HIV-positive controls (Ctrls) based on gating of peripheral blood mononuclear cells (PBMCs) for Lin-negative, HLA-DR ${ }^{\text {low }}$, CD33 ${ }^{+}$, and CD11 $\mathrm{b}^{+}$ cells that were further divided into $\mathrm{CD} 15^{+}$and $\mathrm{CD} 14^{+}$cells. Frequency (percentage of CD15 $5^{+}$cells) of MDSCs in PBMC samples. B-E: CD15 ${ }^{+}$MDSCs in TB/ HIV-co-infected and HIV-positive controls were positively correlated with plasma HIV viral load (C), whereas CD15 ${ }^{+}$MDSCs in TB-infected and TB/HIV-coinfected patients and Ctrls were positively correlated with Mycobacterium tuberculosis antigen load in lymph node tissue (D) but inversely correlated to peripheral CD4 T-cell counts (E) as determined using the Spearman correlation test. $n=8$ TB-infected patients (open circles); $n=7$ TB/HIV-co-infected patients (closed circles); $n=3 \mathrm{HIV}$-negative Ctrls (open squares); $n=4 \mathrm{HIV}$-positive Ctrls (closed squares). ${ }^{* *} P<0.01,{ }^{* *} P<0.001$ (Kruskal-Wallis and Dunn multiple comparisons tests). Ctrl, control.

$\mathrm{CD}_{3} 3^{+} / \mathrm{CD} 11 \mathrm{~b}^{+}$cells were mostly $\mathrm{CD} 15^{+} / \mathrm{CD} 14^{-}$, which is consistent with the phenotype of granulocytic MDSCs (Figure 7A). Indeed, CD15 ${ }^{+}$granulocytic MDSCs were significantly higher in TB/HIV-co-infected patients compared with TB-infected patients $(P=0.02)$ and controls $(P=0.0012)$ (Figure $7 \mathrm{~B}) . \mathrm{CD} 15^{+}$MDSCs were also elevated in HIV-negative TB-infected patients, but this difference was not significant compared with the controls (Figure 7, A and B).

Correlation analyses further showed a positive correlation between the percentage of $\mathrm{CD} 15^{+}$MDSCs in peripheral blood and HIV viral load in plasma (HIV-positive participants; $r=0.77, P=0.0074$ ) or $M$. tuberculosis antigen load in tissue [HIV-positive participants; $r=0.82, P=0.0033$ (data not shown); all study participants; $r=0.71$, $P=0.0002]$ but an inverse correlation between the percentage of $\mathrm{CD}_{15}{ }^{+}$MDSCs and CD4 ${ }^{+} \mathrm{T}$-cell counts in the peripheral circulation [HIV-positive participants; $r=-0.70$, $P=0.0204$ (data not shown); all study participants; $r=-0.55, P=0.0082$ ] (Figure 7, C-E). These results suggested that MDSCs are prevalent in TB/HIV-co-infected patients, both at the site of $M$. tuberculosis infection and in the systemic circulation, and that MDCSs may be associated with progression of TB disease in HIV-positive individuals.

\section{Discussion}

This study demonstrates enhanced chronic inflammation with an accompanying immunosuppressive microenvironment in lymph node granulomas from TB/HIV-co-infected compared with HIV-negative TB-infected patients. TB/HIV co-infection was characterized by poorly formed, necrotic granulomas that displayed a particularly high $M$. tuberculosis antigen expression. Persistent immune activation in TB/HIV co-infection involved not only enhanced production of proinflammatory (IL-6, IFN- $\gamma$, CXCL9, CXCL10, and iNOS) as well as anti-inflammatory (IL-1RA, IL-10, Arg-1, and IDO) mediators but also elevated numbers of myeloid cells with an aberrant phenotype and morphology characteristic of MDSCs in TB-infected lymph nodes. Elevated $\mathrm{IgG}$ responses in TB, and particularly in TB/HIV-co-infected patients, are indicative of chronic immune activation and active TB disease progression. On the other hand, reduced T-cell numbers and low mRNA expression of antimicrobial and cytolytic effector molecules, such as perforin and granulysin, were evident in both HIVnegative TB-infected and TB/HIV-co-infected tissues, whereas immunoregulatory FoxP3 and IDO expression was enhanced. Furthermore, Arg-1 was higher in MDSC-like cells in TB/HIV-co-infected tissues, and granulocytic MDSCs were significantly increased in peripheral blood from TB/HIV-co-infected compared with HIV-negative TB-infected patients. MDSC counts correlated positively with HIV viral load in plasma as well as M. tuberculosis antigen load in tissue but were inversely correlated with peripheral CD4 T-cell counts, which may indicate a role for MDSCs in TB/HIV disease progression.

Pathological features of chronic inflammatory diseases and solid cancers typically include enhanced levels of proinflammatory mediators, such as cytokines, chemokines, and reactive oxygen and nitrogen species. Emerging evidence supports the concept that chronic inflammation promotes local immunosuppression via induction of proinflammatory mediators and the accumulation and activation of immunosuppressive cells. ${ }^{28}$ In line with that view, differentiated macrophages also produce anti-inflammatory cytokines, most notably IL1-RA and IL-10, which are upregulated by proinflammatory mediators and engage in autocrine signaling. ${ }^{29} \mathrm{IL}-10$ reduces HLA-DR expression and antigen presentation in macrophages and inhibits the production of proinflammatory cytokines. ${ }^{30}$ Likewise, 
MDSCs expand during various pathological conditions associated with acute or chronic inflammation. ${ }^{19}$ This paradox may represent both immune exhaustion and active immune inhibition. In TB, defective activation of myeloid cells $^{8,13}$ may contribute to impaired TB immunity by suppression of Th1 effector cells and activation of different pathogenic and Treg cell subsets with a large phenotypic and functional heterogeneity, including expression of immune checkpoint molecules, such as IDO, cytotoxic Tlymphocyte-associated protein (CTLA)-4, programmed death ligand (PD-L)1, and LAG-3. ${ }^{14}$ Although excessive inflammation in TB is harmful and an anti-inflammatory response is required to avoid tissue destruction, local polarization of immunosuppressive subsets with poor antibacterial properties may also delay or prevent the clearance of infection, leading to progressive TB disease. We previously found that high iNOS expression in TB granulomas coincides with impaired Th1 and $\mathrm{CD} 8^{+}$CTL responses but enhance $\mathrm{FoxP}^{+}$Treg cells in patients with active $\mathrm{TB} .^{21}$ The antimicrobial peptides LL-37 and granulysin were reduced in TB lesions from human lung ${ }^{22,23}$ and lymph nodes, ${ }^{21}$ whereas Th2 (IL-4, IL-13, and CCL4) and Treg (FoxP3, CTLA-4, glucocorticoid-induced tumor necrosis factor receptor-related protein, and transforming growth factor- $\beta$ ) responses were enhanced together with excess expression of suppressors of cytokine signaling (SOCS-1 and -3$){ }^{21,25}$ In this study, the localization of Arg- $1^{+}$ MDSC-like cells outside the core of the granuloma in close contact with $\mathrm{CD}^{+}{ }^{+} \mathrm{T}$ cells may partly explain the mixed immune response profile in TB/HIV-co-infected lymph nodes, including induction of both inflammatory and immunosuppressive or anti-inflammatory mediators as determined using mRNA expression analyses.

TB granulomas share many similarities with the microenvironment in solid tumors, including regions of hypoxia and necrosis, extensive fibrosis, and local immunosuppression. Immunosuppressive cell subsets, such as MDSCs, Treg cells, and $\mathrm{T}$ cells, expressing immune checkpoint inhibitors are known to play important roles in tumor immune evasion. MDSCs located within tumor tissue are typically highly immunosuppressive. ${ }^{19,31}$ In particular, both circulating and tumor-infiltrating MDSCs typically express high levels of Arg-1, ${ }^{32,33}$ and high arginase activity at the tumor site correlates with decreased cytokine production and low levels of the T-cell receptor (TCR) CD3 Y-chain. ${ }^{34}$ In patients with non-small cell lung cancer, granulocytic MDCSs in peripheral blood were found to be inversely correlated with $\mathrm{CD} 8^{+} \mathrm{T}$-cell frequencies. ${ }^{35}$ In those with renal or lung carcinoma, either depletion of MDSCs or inhibition of Arg-1 was found to

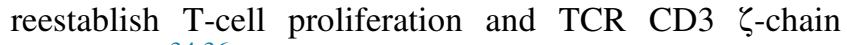
expression. ${ }^{34,36}$ Recent studies also demonstrated that MDSCs mainly exert immunosuppressive functions through the induction of Arg-1 and L-arginine depletion. ${ }^{37-39}$ Circulating MDSCs in patients with gastric cancer expressed Arg-1 that was associated with decreased
$\mathrm{CD}^{+}$T-cell proliferation as well as with reduced IFN- $\gamma$ production and granzyme B secretion from those cells. ${ }^{40}$ Similarly, elevated levels of MDCSs in patients with chronic hepatitis $\mathrm{C}$ infection are associated with a downregulation of the TCR $\zeta$-chain on $\mathrm{CD}^{+} \mathrm{T}$ cells. ${ }^{41}$ MDSCs in this inflammatory environment produce Arg-1 and increased Arg- $1^{+}$cells in the liver were closely associated with tissue pathology in patients with chronic hepatitis. ${ }^{41}$ Recently, it was also reported that monocytic MDSCs expanded in blood from patients with coronavirus disease 2019 and were strongly associated with disease severity, including suppressed $\mathrm{T}$-cell proliferation and IFN- $\gamma$ production as well as down-regulated expression of the CD3 $\zeta$-chain, partly via an Arg-1-dependent mechanism. ${ }^{42}$

Despite extensive evidence of MDSCs in different types of cancer, relatively few studies have investigated these cells in either HIV or TB infection, and assessments of MDSCs at the site of infection are scarce. In HIV infection, the original findings suggest that peripheral granulocytic MDSCs were increased in highly active antiretroviral therapy-naive HIV-positive patients but rapidly declined after the start of therapy. ${ }^{9}$ Similar to the results of this study, MDSC numbers in blood showed a positive correlation with HIV viral loads and a negative correlation with $\mathrm{CD}^{+}{ }^{+}$T-cell counts. ${ }^{9}$ Enhanced levels of immunosuppressive monocytic MDSC have also been found in HIVinfected patients despite 2 years of effective antiretroviral therapy. ${ }^{43}$ Uncontrolled HIV replication has been observed to result in persistent immune activation, including elevated IL-6 levels, that mediates an expansion of MDSC numbers in HIV-infected patients. ${ }^{44,45}$ Furthermore, HIVinduced MDSCs may increase the expression of inhibitory mediators, including IL-10 and PD-L1 protein, ${ }^{46}$ restrict proliferation of effector $\mathrm{T}$ cells, and instead promote the differentiation of FoxP3 ${ }^{+}$Treg cells in T-cell cocultures. $9,44,46$ Accordingly, granulocytic MDSCs expressing PD-L1 protein could inhibit both proliferation and IFN- $\gamma$ production of $\mathrm{CD}^{+} \mathrm{T}$ cells from HIV-infected patients. ${ }^{47}$ Another report observed an inverse correlation between granulocytic MDSCs and expression of the TCR CD3 $\zeta$-chain in HIV-infected patients that was restored on in vitro depletion of MDSCs. ${ }^{48}$

In human TB, MDCSs that suppress T-cell functions were initially described in the peripheral blood and in pleural fluid from patients with active TB. ${ }^{8}$ MDSC levels in patients with active TB were comparable to those in patients with cancer and contributed to reduced Th1 cytokine expression, whereas proinflammatory cytokines, such as IL-1 $\beta$, IL-6, IL-8, and monocyte chemoattractant protein-1, were increased. ${ }^{8}$ High frequencies of granulocytic MDSCs have been observed in both peripheral blood and bronchoalveolar lavage specimens from patients with active pulmonary TB that suppressed T-cell proliferation, whereas MDSC were rapidly reduced on effective anti-TB treatment. ${ }^{49}$ Increased MDSC levels in peripheral blood 
from TB-infected children have also been linked to heightened IDO and Arg-1 levels in matched plasma samples. ${ }^{50}$ Furthermore, in vitro generated MDSCs promote $M$. tuberculosis replication by altering granuloma structure and stability and shift the balance of soluble mediators at the site of infection toward suppressive and regulatory cytokines, including a high release of IL-10 and IL- $6,{ }^{51}$ which is consistent with the mRNA profiling data in the current study. MDSCs from HIV-infected individuals have also demonstrated defective innate immunity to $M$. tuberculosis and exhibited a higher intracellular replication of $M$. tuberculosis compared with HLA-DR ${ }^{\text {hi }}$ myeloid subsets. ${ }^{52}$ This finding is consistent with an enhanced $M$. tuberculosis antigen expression in TB/HIV-co-infected lymph nodes as well as elevated $M$. tuberculosis antigen load in TB/HIV granulomas compared with HIV-negative TB, which may imply that HIV contributes to an immune cell dysfunction locally in the tissue that will make macrophage subsets more permissive to intracellular growth of M. tuberculosis. In turn, an enhanced bacterial replication may foster the expansion of MDSCs in TB/HIV-co-infected patients.

In TB-HIV-co-infected lymph node granulomas, IDO was mostly expressed in $\mathrm{CD} 8^{+}$macrophages, which is consistent with previous findings. ${ }^{53}$ In contrast, iNOS was abundantly expressed around the $\mathrm{CD} 68^{+}$core of the granulomas, although we were not able to fully characterize the phenotype of iNOS-producing cells. Immunosuppressive MDSCs from patients with pulmonary TB correlated with depleted serum levels of L-arginine but increased levels of NO, suggesting that MDSCs may exert their suppression by enhanced iNOS activity. ${ }^{49}$ In line with this finding, BCG vaccination of mice resulted in infiltration of NO-producing MDSCs into the skin that were unable to kill BCG bacteria but instead impaired T-cell priming in the draining lymph nodes. ${ }^{54}$ iNOS-expressing cells with immunosuppressive functions resembling monocytic MDSCs have also been identified in HIV and simian immunodeficiency virus infection. ${ }^{55}$ Because NO production by myeloid cells can contribute to suppressive $\mathrm{T}$-cell responses, it is possible that iNOS-expressing cells observed in TB/HIV-co-infected lymph node tissue also represent a subset of suppressive myeloid cells.

Recent evidence suggests that pharmacologic exhaustion of MDSCs with the small-molecule inhibitor tasquinimod resulted in reduced $\mathrm{FoxP}^{+}$Treg cells but enhanced $\mathrm{CD} 8^{+}$ effector $\mathrm{T}$ cells in the lung of $M$. tuberculosis-infected mice, and tasquinimod-mediated depletion of MDSCs also enhanced $M$. tuberculosis clearance in the lungs. ${ }^{56}$ In addition, tasquinimod impaired the formation of organized TB granulomas that contain cores of neutrophils with MDSC-like properties. ${ }^{57}$ In the microenvironment of solid tumors, tasquinimod treatment was also observed to decrease Arg-1 and CD206 mRNA expression in macrophages, indicating a shift from M2-like myeloid cells to the M1 phenotype. ${ }^{58}$ In a mouse lung tumor model, inhibition of Arg-1 in myeloid cells diminished growth of established tumors by increasing T-cell infiltration into the tumor tissue, which was accompanied by a significant increase in the $\mathrm{CD} 8 / \mathrm{Foxp} 3$ ratio and IFN- $\gamma$ production in T cells isolated from the tumors. ${ }^{59}$ Similarly, another small-molecule inhibitor of Arg-1 reversed myeloid cell-mediated immunosuppression and restored $\mathrm{T}$-cell proliferation in different solid tumors, supporting the value of Arg-1 as an immunomodulatory target. ${ }^{60}$ These reports suggest that inactivation or depletion of MDSC subsets or a shift toward proinflammatory myeloid cells might have potential in effective host-directed therapy that could be a powerful adjunct to conventional antibiotic therapy in chronic TB and TB-HIV co-infection.

\section{Acknowledgments}

We thank Elisabeth Henriksson for excellent technical assistance on the paraffin-embedded staining protocol and Anette Hofmann for her help with confocal microscopy, Alemayehu Kifle at the Armauer Hansen Research Institute for transportation and storage of the patient samples, and $\mathrm{Sr}$. Alemtsehay, surgical nurse at the Department of Surgery, Tikur Anbessa Specialized Hospital, for recruitment and follow-up of the patients. Finally, we acknowledge the patients who participated in this study.

\section{Author Contributions}

S.A., B.L., A.B., G.Ad., M.S., and S.B. designed and initiated the study, including preparation of study protocols and ethical approval; S.B. provided financial support for the study; S.A. was involved in all aspects of the clinical and laboratory work and coordinated the study with support from the co-authors as follows: B.L. recruited and managed all the patients; G.As. analyzed chest radiographic findings and contributed to patient diagnosis; M.Z. and M.C. participated in patient sample preparation and in vitro cell culture work; J.R.M. contributed to flow cytometry analyses and enzyme-linked immunosorbent assay; J.S. and S.R. participated in the confocal microscopy and flow cytometry analyses; A.R. performed quantitative PCR analyses; A.M. assisted with immunohistochemistry; S.B. and S.A. wrote, edited, and revised the manuscript with input from the other authors; all authors approved the final version of manuscript.

\section{Supplemental Data}

Supplemental material for this article can be found at http://doi.org/10.1016/j.ajpath.2021.12.013.

\section{References}

1. World Health Organization: WHO Global Tuberculosis Report 2020 Geneva, World Health Organization, 2020 
2. Brighenti S, Andersson J: Local immune responses in human tuberculosis: learning from the site of infection. J Infect Dis 2012, 205(Suppl 2):S316-S324

3. Flynn JL, Chan J, Lin PL: Macrophages and control of granulomatous inflammation in tuberculosis. Mucosal Immunol 2011, 4: $271-278$

4. Saunders BM, Cooper AM: Restraining mycobacteria: role of granulomas in mycobacterial infections. Immunol Cell Biol 2000, 78: 334-341

5. de Noronha AL, Bafica A, Nogueira L, Barral A, Barral-Netto M: Lung granulomas from Mycobacterium tuberculosis/HIV-1 coinfected patients display decreased in situ TNF production. Pathol Res Pract 2008, 204:155-161

6. Lawn SD, Butera ST, Shinnick TM: Tuberculosis unleashed: the impact of human immunodeficiency virus infection on the host granulomatous response to Mycobacterium tuberculosis. Microbes Infect 2002, 4:635-646

7. Gabrilovich DI: Myeloid-derived suppressor cells. Cancer Immunol Res 2017, 5:3-8

8. du Plessis N, Loebenberg L, Kriel M, von Groote-Bidlingmaier F, Ribechini E, Loxton AG, van Helden PD, Lutz MB, Walzl G: Increased frequency of myeloid-derived suppressor cells during active tuberculosis and after recent mycobacterium tuberculosis infection suppresses T-cell function. Am J Respir Crit Care Med 2013, 188: $724-732$

9. Vollbrecht T, Stirner R, Tufman A, Roider J, Huber RM, Bogner JR, Lechner A, Bourquin C, Draenert R: Chronic progressive HIV-1 infection is associated with elevated levels of myeloid-derived suppressor cells. AIDS 2012, 26:F31-F37

10. Bronte V, Brandau S, Chen SH, Colombo MP, Frey AB, Greten TF, Mandruzzato S, Murray PJ, Ochoa A, Ostrand-Rosenberg S, Rodriguez PC, Sica A, Umansky V, Vonderheide RH, Gabrilovich DI: Recommendations for myeloid-derived suppressor cell nomenclature and characterization standards. Nat Commun 2016, 7:12150

11. Magcwebeba T, Dorhoi A, du Plessis N: The emerging role of myeloid-derived suppressor cells in tuberculosis. Front Immunol 2019, 10:917

12. Yang B, Wang X, Ren X: Amino acid metabolism related to immune tolerance by MDSCs. Int Rev Immunol 2012, 31:177-183

13. Thomas AC, Mattila JT: "Of mice and men": arginine metabolism in macrophages. Front Immunol 2014, 5:479

14. Brighenti S, Joosten SA: Friends and foes of tuberculosis: modulation of protective immunity. J Intern Med 2018

15. Rath M, Muller I, Kropf P, Closs EI, Munder M: Metabolism via arginase or nitric oxide synthase: two competing arginine pathways in macrophages. Front Immunol 2014, 5:532

16. Mills CD, Ley K: M1 and M2 macrophages: the chicken and the egg of immunity. J Innate Immun 2014, 6:716-726

17. Munder M, Schneider H, Luckner C, Giese T, Langhans CD, Fuentes JM, Kropf P, Mueller I, Kolb A, Modolell M, Ho AD: Suppression of T-cell functions by human granulocyte arginase. Blood 2006, 108:1627-1634

18. Fallarino F, Grohmann U, You S, McGrath BC, Cavener DR, Vacca C, Orabona C, Bianchi R, Belladonna ML, Volpi C, Santamaria P, Fioretti MC, Puccetti P: The combined effects of tryptophan starvation and tryptophan catabolites down-regulate $\mathrm{T}$ cell receptor zeta-chain and induce a regulatory phenotype in naive $\mathrm{T}$ cells. J Immunol 2006, 176:6752-6761

19. Gabrilovich DI, Nagaraj S: Myeloid-derived suppressor cells as regulators of the immune system. Nat Rev Immunol 2009, 9: 162-174

20. Deveci HS, Kule M, Kule ZA, Habesoglu TE: Diagnostic challenges in cervical tuberculous lymphadenitis: a review. North Clin Istanb 2016, 3:150-155

21. Rahman S, Gudetta B, Fink J, Granath A, Ashenafi S, Aseffa A, Derbew M, Svensson M, Andersson J, Brighenti SG:
Compartmentalization of immune responses in human tuberculosis: few CD8+ effector T cells but elevated levels of FoxP3+ regulatory t cells in the granulomatous lesions. Am J Pathol 2009, 174: $2211-2224$

22. Rahman S, Rehn A, Rahman J, Andersson J, Svensson M, Brighenti S: Pulmonary tuberculosis patients with a vitamin D deficiency demonstrate low local expression of the antimicrobial peptide LL-37 but enhanced FoxP3 + regulatory T cells and IgG-secreting cells. Clin Immunol 2015, 156:85-97

23. Andersson J, Samarina A, Fink J, Rahman S, Grundstrom S: Impaired expression of perforin and granulysin in CD8 $+\mathrm{T}$ cells at the site of infection in human chronic pulmonary tuberculosis. Infect Immun 2007, 75:5210-5222

24. Wangoo A, Johnson L, Gough J, Ackbar R, Inglut S, Hicks D, Spencer Y, Hewinson G, Vordermeier M: Advanced granulomatous lesions in Mycobacterium bovis-infected cattle are associated with increased expression of type I procollagen, gammadelta $(\mathrm{WC} 1+) \mathrm{T}$ cells and CD 68+ cells. J Comp Pathol 2005, 133:223-234

25. Ashenafi S, Aderaye G, Bekele A, Zewdie M, Aseffa G, Hoang AT, Carow B, Habtamu M, Wijkander M, Rottenberg M, Aseffa A, Andersson J, Svensson M, Brighenti S: Progression of clinical tuberculosis is associated with a Th2 immune response signature in combination with elevated levels of SOCS3. Clin Immunol 2014, 151:84-99

26. Cassetta L, Baekkevold ES, Brandau S, Bujko A, Cassatella MA, Dorhoi A, Krieg C, Lin A, Lore K, Marini O, Pollard JW, Roussel M, Scapini P, Umansky V, Adema GJ: Deciphering myeloid-derived suppressor cells: isolation and markers in humans, mice and nonhuman primates. Cancer Immunol Immunother 2019, 68:687-697

27. Bayik D, Lauko AJ, Roversi GA, Serbinowski E, AcevedoMoreno LA, Lanigan C, Orujov M, Lo A, Alban TJ, Kim A, Silver DJ, Nagy LE, Brown JM, Allende DS, Aucejo FN, Lathia JD: Hepatobiliary malignancies have distinct peripheral myeloid-derived suppressor cell signatures and tumor myeloid cell profiles. Sci Rep 2020, 10:18848

28. Wang D, DuBois RN: Immunosuppression associated with chronic inflammation in the tumor microenvironment. Carcinogenesis 2015, 36:1085-1093

29. Kinne RW, Brauer R, Stuhlmuller B, Palombo-Kinne E, Burmester GR: Macrophages in rheumatoid arthritis. Arthritis Res 2000, 2:189-202

30. Isomaki P, Luukkainen R, Saario R, Toivanen P, Punnonen J: Interleukin-10 functions as an antiinflammatory cytokine in rheumatoid synovium. Arthritis Rheum 1996, 39:386-395

31. Movahedi K, Guilliams M, Van den Bossche J, Van den Bergh R, Gysemans C, Beschin A, De Baetselier P, Van Ginderachter JA: Identification of discrete tumor-induced myeloid-derived suppressor cell subpopulations with distinct $\mathrm{T}$ cell-suppressive activity. Blood 2008, 111:4233-4244

32. Rodriguez PC, Ochoa AC, Al-Khami AA: Arginine metabolism in myeloid cells shapes innate and adaptive immunity. Front Immunol 2017, 8:93

33. Ren W, Zhang X, Li W, Feng Q, Feng H, Tong Y, Rong H, Wang W, Zhang D, Zhang Z, Tu S: Circulating and tumor-infiltrating arginase 1-expressing cells in gastric adenocarcinoma patients were mainly immature and monocytic Myeloid-derived suppressor cells. Sci Rep 2020, 10:8056

34. Zea AH, Rodriguez PC, Atkins MB, Hernandez C, Signoretti S, Zabaleta J, McDermott D, Quiceno D, Youmans A, O'Neill A, Mier J, Ochoa AC: Arginase-producing myeloid suppressor cells in renal cell carcinoma patients: a mechanism of tumor evasion. Cancer Res 2005, 65:3044-3048

35. Liu CY, Wang YM, Wang CL, Feng PH, Ko HW, Liu YH, Wu YC, Chu Y, Chung FT, Kuo CH, Lee KY, Lin SM, Lin HC, Wang CH, Yu CT, Kuo HP: Population alterations of L-arginase- and inducible nitric oxide synthase-expressed CD11b+/CD14(-)/CD15+/CD33+ myeloid-derived suppressor cells and $\mathrm{CD} 8+\mathrm{T}$ lymphocytes in 
patients with advanced-stage non-small cell lung cancer. J Cancer Res Clin Oncol 2010, 136:35-45

36. Rodriguez PC, Quiceno DG, Zabaleta J, Ortiz B, Zea AH, Piazuelo MB, Delgado A, Correa P, Brayer J, Sotomayor EM, Antonia S, Ochoa JB, Ochoa AC: Arginase I production in the tumor microenvironment by mature myeloid cells inhibits T-cell receptor expression and antigen-specific T-cell responses. Cancer Res 2004, 64:5839-5849

37. Kong YY, Fuchsberger M, Xiang SD, Apostolopoulos V, Plebanski M: Myeloid derived suppressor cells and their role in diseases. Curr Med Chem 2013, 20:1437-1444

38. Rodriguez PC, Ochoa AC: Arginine regulation by myeloid derived suppressor cells and tolerance in cancer: mechanisms and therapeutic perspectives. Immunol Rev 2008, 222:180-191

39. Raber P, Ochoa AC, Rodriguez PC: Metabolism of L-arginine by myeloid-derived suppressor cells in cancer: mechanisms of $\mathrm{T}$ cell suppression and therapeutic perspectives. Immunol Invest 2012, 41: 614-634

40. Mao FY, Zhao YL, Lv YP, Teng YS, Kong H, Liu YG, Wu XL, Hao CJ, Chen W, Duan MB, Han B, Ma Q, Wang TT, Peng LS, Zhang JY, Cheng P, Su CY, Fu XL, Zou QM, Guo G, Guo XL, Zhuang Y: CD45(+)CD33(low)CD11b(dim) myeloid-derived suppressor cells suppress CD8(+) $\mathrm{T}$ cell activity via the IL-6/IL-8arginase I axis in human gastric cancer. Cell Death Dis 2018, 9:763

41. Zeng QL, Yang B, Sun HQ, Feng GH, Jin L, Zou ZS, Zhang Z, Zhang JY, Wang FS: Myeloid-derived suppressor cells are associated with viral persistence and downregulation of TCR zeta chain expression on $\mathrm{CD} 8(+) \mathrm{T}$ cells in chronic hepatitis $\mathrm{C}$ patients. Mol Cells 2014, 37:66-73

42. Falck-Jones S, Vangeti S, Yu M, Falck-Jones R, Cagigi A, Badolati I, Osterberg B, Lautenbach MJ, Ahlberg E, Lin A, Lepzien R, Szurgot I, Lenart K, Hellgren F, Maecker H, Salde J, Albert J, Johansson N, Bell M, Lore K, Farnert A, Smed-Sorensen A: Functional monocytic myeloid-derived suppressor cells increase in blood but not airways and predict COVID-19 severity. J Clin Invest 2021, 131:e144734

43. Qin A, Cai W, Pan T, Wu K, Yang Q, Wang N, Liu Y, Yan D, Hu F, Guo P, Chen X, Chen L, Zhang H, Tang X, Zhou J: Expansion of monocytic myeloid-derived suppressor cells dampens $\mathrm{T}$ cell function in HIV-1-seropositive individuals. J Virol 2013, 87:1477-1490

44. Garg A, Spector SA: HIV type 1 gp120-induced expansion of myeloid derived suppressor cells is dependent on interleukin 6 and suppresses immunity. J Infect Dis 2014, 209:441-451

45. Garg A, Trout R, Spector SA: Human immunodeficiency virus type-1 myeloid derived suppressor cells inhibit cytomegalovirus inflammation through interleukin-27 and B7-H4. Sci Rep 2017, 7: 44485

46. Wang L, Zhao J, Ren JP, Wu XY, Morrison ZD, Elgazzar MA, Ning SB, Moorman JP, Yao ZQ: Expansion of myeloid-derived suppressor cells promotes differentiation of regulatory $\mathrm{T}$ cells in HIV-1+ individuals. AIDS 2016, 30:1521-1531

47. Zhang ZN, Yi N, Zhang TW, Zhang LL, Wu X, Liu M, Fu YJ, He SJ, Jiang YJ, Ding HB, Chu ZX, Shang H: Myeloid-derived suppressor cells associated with disease progression in primary HIV infection: PD-L1 blockade attenuates inhibition. J Acquir Immune Defic Syndr 2017, 76:200-208

48. Tumino N, Turchi F, Meschi S, Lalle E, Bordoni V, Casetti R, Agrati C, Cimini E, Montesano C, Colizzi V, Martini F, Sacchi A: In HIV-positive patients, myeloid-derived suppressor cells induce T-cell anergy by suppressing CD3zeta expression through ELF-1 inhibition. AIDS 2015, 29:2397-2407
49. El Daker S, Sacchi A, Tempestilli M, Carducci C, Goletti D, Vanini V, Colizzi V, Lauria FN, Martini F, Martino A: Granulocytic myeloid derived suppressor cells expansion during active pulmonary tuberculosis is associated with high nitric oxide plasma level. PLoS One 2015, 10:e0123772

50. Du Plessis N, Jacobs R, Gutschmidt A, Fang Z, van Helden PD, Lutz MB, Hesseling AC, Walzl G: Phenotypically resembling myeloid derived suppressor cells are increased in children with HIV and exposed/infected with Mycobacterium tuberculosis. Eur J Immunol 2017, 47:107-118

51. Agrawal N, Streata I, Pei G, Weiner J, Kotze L, Bandermann S, Lozza L, Walzl G, du Plessis N, Ioana M, Kaufmann SHE, Dorhoi A: Human monocytic suppressive cells promote replication of Mycobacterium tuberculosis and alter stability of in vitro generated granulomas. Front Immunol 2018, 9:2417

52. Garg A, Spector SA: Myeloid derived suppressor cells (MDSC) from HIV-infected individuals are defective in innate immunity to Mycobacterium tuberculosis thus increasing the risk of tuberculosis. J Immunol 2019, 202(1 Supplement):62.13

53. Gautam US, Foreman TW, Bucsan AN, Veatch AV, Alvarez X, Adekambi T, Golden NA, Gentry KM, Doyle-Meyers LA, RussellLodrigue KE, Didier PJ, Blanchard JL, Kousoulas KG, Lackner AA, Kalman D, Rengarajan J, Khader SA, Kaushal D, Mehra S: In vivo inhibition of tryptophan catabolism reorganizes the tuberculoma and augments immune-mediated control of Mycobacterium tuberculosis. Proc Natl Acad Sci U S A 2018, 115:E62-E71

54. Martino A, Badell E, Abadie V, Balloy V, Chignard M, Mistou MY, Combadiere B, Combadiere C, Winter N: Mycobacterium bovis bacillus Calmette-Guerin vaccination mobilizes innate myeloid-derived suppressor cells restraining in vivo $\mathrm{T}$ cell priming via IL-1Rdependent nitric oxide production. J Immunol 2010, 184:2038-2047

55. Gama L, Shirk EN, Russell JN, Carvalho KI, Li M, Queen SE, Kalil J, Zink MC, Clements JE, Kallas EG: Expansion of a subset of CD14highCD16negCCR2low/neg monocytes functionally similar to myeloid-derived suppressor cells during SIV and HIV infection. J Leukoc Biol 2012, 91:803-816

56. Gupta S, Krug S, Pokkali S, Leanderson T, Isaacs JT, Srikrishna G, Bishai WR: Pharmacologic exhaustion of suppressor cells with tasquinimod enhances bacterial clearance during tuberculosis. Am J Respir Crit Care Med 2019, 199:386-389

57. Yoshioka Y, Mizutani T, Mizuta S, Miyamoto A, Murata S, Ano T, Ichise H, Morita D, Yamada H, Hoshino Y, Tsuruyama T, Sugita M: Neutrophils and the S100A9 protein critically regulate granuloma formation. Blood Adv 2016, 1:184-192

58. Olsson A, Nakhle J, Sundstedt A, Plas P, Bauchet AL, Pierron V, Bruetschy L, Deronic A, Torngren M, Liberg D, Schmidlin F, Leanderson T: Tasquinimod triggers an early change in the polarization of tumor associated macrophages in the tumor microenvironment. J Immunother Cancer 2015, 3:53

59. Miret JJ, Kirschmeier P, Koyama S, Zhu M, Li YY, Naito Y, Wu M, Malladi VS, Huang W, Walker W, Palakurthi S, Dranoff G, Hammerman PS, Pecot CV, Wong KK, Akbay EA: Suppression of myeloid cell arginase activity leads to therapeutic response in a NSCLC mouse model by activating anti-tumor immunity. J Immunother Cancer 2019, 7:32

60. Steggerda SM, Bennett MK, Chen J, Emberley E, Huang T, Janes JR, Li W, MacKinnon AL, Makkouk A, Marguier G, Murray PJ, Neou S, Pan A, Parlati F, Rodriguez MLM, Van de Velde LA, Wang T, Works M, Zhang J, Zhang W, Gross MI: Inhibition of arginase by CB-1158 blocks myeloid cell-mediated immune suppression in the tumor microenvironment. J Immunother Cancer 2017, 5:101 